\title{
A three dimensional integral equation approach for fluids under confinement: Argon in zeolites
}

\author{
Enrique Lomba, ${ }^{\text {a) }}$ Cecilia Bores, Vicente Sánchez-Gil, and Eva G. Noya \\ Instituto de Química Física Rocasolano, CSIC, Serrano 119, E-28006 Madrid, Spain
}

(Received 15 July 2015; accepted 8 October 2015; published online 23 October 2015)

In this work, we explore the ability of an inhomogeneous integral equation approach to provide a full three dimensional description of simple fluids under conditions of confinement in porous media. Explicitly, we will consider the case of argon adsorbed into silicalite-1, silicalite-2, and an all-silica analogue of faujasite, with a porous structure composed of linear (and zig-zag in the case of silicalite-1) channels of 5-8 $\AA$ diameter. The equation is based on the three dimensional Ornstein-Zernike approximation proposed by Beglov and Roux [J. Chem. Phys. 103, 360 (1995)] in combination with the use of an approximate fluid-fluid direct correlation function furnished by the replica Ornstein-Zernike equation with a hypernetted chain closure. Comparison with the results of grand canonical Monte Carlo/molecular dynamics simulations evidences that the theory provides an accurate description for the three dimensional density distribution of the adsorbed fluid, both at the level of density profiles and bidimensional density maps across representative sections of the porous material. In the case of very tight confinement (silicalite- 1 and silicalite-2), solutions at low temperatures could not be found due to convergence difficulties, but for faujasite, which presents substantially larger channels, temperatures as low as $77 \mathrm{~K}$ are accessible to the integral equation. The overall results indicate that the theoretical approximation can be an excellent tool to characterize the microscopic adsorption behavior of porous materials. (C) 2015 AIP Publishing LLC. [http://dx.doi.org/10.1063/1.4934230]

\section{INTRODUCTION}

For the last two decades we have witnessed a growing interest on the explicit three-dimensional spatial description of the structure of fluids in the vicinity of large molecules, mainly due to its relevance for the solvation mechanisms of biomolecular systems. ${ }^{1}$ The knowledge of the solvent structure inside specific sites within a biomolecule is a key for the understanding of many biological processes, such as the catalytic activity of enzymes. It is in this context where the interest on the solution of the full three dimensional OrnsteinZernike (3DOZ) equation was revived already 20 years ago when Beglov and Roux ${ }^{2}$ first solved the 3DOZ in the Hypernetted Chain (HNC) approximation for a system of a single molecule (solute) of arbitrary shape immersed in an atomic solvent. This approach is in fact a "singlet" approach, ${ }^{3}$ in the sense that it provides a density profile of the solvent in the external field of the solute, with the approximation that solvent-solvent correlations are averaged over the inhomogeneity. These correlations were approximated by those of the bulk solvent. This was known as the Henderson-AbrahamBarker equation when dealing with atomic liquids. ${ }^{4}$ Additionally, the approach of Beglov and Roux can also be interpreted in terms of an inhomogeneous HNC equation as derived from the Density Functional theory (DFT) approach ${ }^{6,7}$ of Ramakrishnan and Yussouff, ${ }^{5}$ which, in turn, opens an avenue for further improvements of the original formulation.

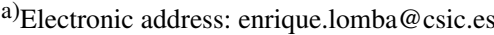

The work of Ref. 2 has been later extended to molecular liquids, both polar and non-polar ${ }^{8-10}$ and it turned out to be particularly successful when applying the Reference Interact Site Model (RISM) approach ${ }^{3,11,12}$ to describe solutesolvent and solvent-solvent correlations. The exact treatment of Coulomb interactions within this class of three dimensional approximations has also been recently formulated by Perkyns et al. ${ }^{1}$

In close connection with this, detailed information on the spatial distribution of confined fluids is also of paramount importance in many processes of industrial interest, such as gas storage or catalysis. Very specially when dealing with functionalized adsorbents, ${ }^{13}$ the knowledge of the adsorbate distribution within the adsorbent is essential. In fact, zeolite design for catalytic applications has also been inspired by the processes of molecular recognition characteristic of enzymes, ${ }^{14}$ where solvation is an essential element. Experimental techniques such as FTIR and NMR spectroscopy, ${ }^{15,16}$ or neutron scattering ${ }^{17,18}$ provide very valuable information in this context, but if one desires a detailed three dimensional map of the confined fluid structure, the use of computer simulation is unavoidable. The calculation of density maps, particularly in regions where the sampling is poor due to problems of accessibility might turn into a computationally lengthy process. ${ }^{19}$ Therefore, the development of theoretical tools capable of describing explicitly and accurately the three dimensional structure of a fluid confined in porous material is of primary interest. Already in the work of Beglov and Roux, ${ }^{2}$ these authors computed the density profile of a simple fluid adsorbed into a crude model of zeolite solving the 3DOZ-HNC equation and 
using as an approximation for the fluid-fluid direct correlation function (dcf) that of the bulk fluid. More recently, two of the authors in collaboration with $\mathrm{Kahl}^{19}$ revisited the approach of Beglov and Roux to describe the structure of a fluid inclusion into a model of controlled pore glass in two-dimensions. Since the model in question could be thought as a partly quenched mixture, the authors resorted to the use of the Replica Ornstein Zernike (ROZ) approach ${ }^{20-22}$ in order to approximate the fluidfluid dcf. The use of a direct correlation function corresponding to a confined fluid averaged over the porous matrix disorder improved upon the simple bulk fluid approximation, which although suitable for solvation problems, is of limited validity under conditions of tight confinement. ${ }^{19}$ The results presented in Ref. 19 for fluids interacting via short range attractive-long range repulsive (SALR) and Lennard-Jones (LJ) potentials indicate that the combination of the 3DOZ-HNC and ROZHNC equations is a promising alternative to describe explicitly the spatial distribution of fluids under conditions of confinement in the nanometer regime.

As a natural extension of the work of Ref. 19, we will here present the solution of the 3DOZ-HNC equation for a simple fluid, argon modeled as a one-site LJ fluid, adsorbed into three zeolite models, namely, silicalite-1 (all silica analogue of the ZSM-5 zeolite, framework type MFI), silicalite-2 (all silica analogue of the ZSM-11 zeolite, framework type MEL) and an all silica model of faujasite (framework type FAU). Both MFI and MEL zeolites are composed of 4-, 5-, and 6-membered rings linked to form a system of channels with 10-membered ring openings. ${ }^{23}$ In MFI, the channels are a combination of linear and zig-zag, while in MEL-type zeolites, all the channels are linear. Their channels are typically in the range of 5.4$5.6 \AA$ wide. The channels in faujasite are formed by 12 membered rings and are also linear, being considerably wider (7.4 $\AA$ ) and intersecting to form a cavity of $12 \AA$ of diameter. In contrast to our previous work, ${ }^{19}$ the adsorbent material is now formed by a regular network of interconnected channels. Even if the system composed by the fluid and the adsorbent could still be thought as a partly quenched mixture, ${ }^{24}$ there is no room for averaging over disorder now. We are in the presence of a regular and highly inhomogeneous confining medium, so the applicability of the ROZ equations to this system is in principle questionable. Nonetheless, here we will still consider the zeolite as the quenched component in the ROZ equations, and hence, its exact structure factor will be used to describe the corresponding adsorbent-adsorbent correlations. We will see then that the average fluid-fluid and fluid-adsorbent correlation functions provided by the ROZ-HNC equations are qualitatively correct when compared with simulation results. Consequently, in the context of the 3DOZ-HNC equation, the use of ROZ-HNC fluid-fluid direct correlation functions will be a better approximation than employing bulk fluid correlations. With this, we have in our hands the required elements to solve the 3DOZ-HNC approximation for our model of Ar adsorbed into zeolites. We will compare density maps across relevant sections of the zeolites and density profiles along the most significant directions calculated by means of our theoretical approach and computer simulation. We will see that the theory is able to provide a detailed picture of the adsorbed fluid, illustrating those locations within the porous structure where adsorbed particles are most likely to be found.

The rest of the paper is organized as follows. In Sec. II, we briefly introduce our model for the zeolite and the adsorbed fluid. The 3DOZ-HNC approach is sketched in Section III together with a short summary of the main equations of the ROZ-HNC theory. Our most significant results are commented upon in Section IV. Concluding remarks and future prospects are collected in Section V.

\section{THE MODEL}

Our system is composed of Ar atoms which interact via a $\mathrm{LJ}$ potential of the form

$$
u_{A r A r}(r)=4 \varepsilon_{A r A r}\left[\left(\frac{\sigma_{A r A r}}{r}\right)^{12}-\left(\frac{\sigma_{A r A r}}{r}\right)^{6}\right],
$$

with $\varepsilon_{A r A r} / k_{B}=124.07 \mathrm{~K}$ and $\sigma_{A r A r}=3.38 \AA$, and $k_{B}$ being Boltzmann's constant as usual. ${ }^{25}$ The structure of the three zeolites in question is illustrated in Figure 1. For our calculations, we have used a super-cell of $2 \times 2 \times 3$ unit cells for the MFI and MEL frameworks and one of $2 \times 2 \times 2$ unit cells for the FAU framework. Structural data for these zeolites are taken from Refs. 26-28. For the zeolite-Ar interaction, we will use the model of García-Pérez et al. ${ }^{25}$ that is, a Lennard-Jones interaction with $\varepsilon_{O A r} / k_{B}=107.69 \mathrm{~K}$ and $\sigma_{O A r}=3.15 \AA$. As it is customary when modeling adsorption in zeolites, if charges are not taken into account, the interaction between $\mathrm{Ar}$ and $\mathrm{Si}$ atoms is neglected, being the latter completely embedded within the oxygen tetrahedra. In the case of the faujasite framework, one must take into account that in each unit cell there are eight inaccessible cavities, large enough to host an Ar atom. Obviously, these cavities will not participate in the adsorption process, and this must be borne in mind both when running grand canonical Monte Carlo (GCMC) simulations or when building the matrix-adsorbate potential for the solution of the 3DOZ equation.

For computational efficiency, we have truncated and shifted the interactions at $R_{c}=3 \sigma_{A r A r}$. In what follows, we will designate the oxygen atoms by the subscript (or superscript) 0 (the matrix or adsorbent) and Ar atom by subscript (superscript) 1 (fluid or adsorbate).

\section{THEORY}

Following our previous work in two dimensions, ${ }^{19}$ an explicit description of the structure of a fluid confined in a porous medium, such as a zeolite, can be achieved by means of an approximation to the full three dimensional solution of the Ornstein-Zernike (OZ) equation according to the prescription of Beglov and Roux. ${ }^{2}$ Despite the fact that our porous medium is topologically ordered, we will also here resort to the replica Ornstein-Zernike formalism ${ }^{20-22}$ in order to provide a reasonable approximation for the confined fluid direct correlation function averaged over the inhomogeneities of the medium. In Sec. III A, the ROZ equations are summarized, and the details of the solution procedure of the 3DOZ equation are commented upon in Sec. III B. 


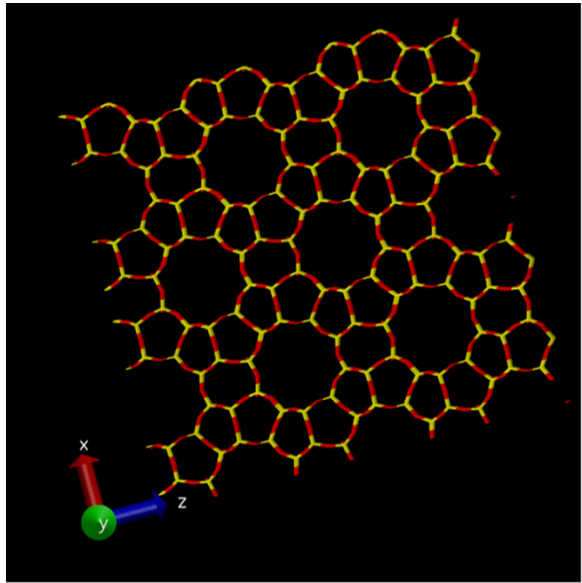

(a)

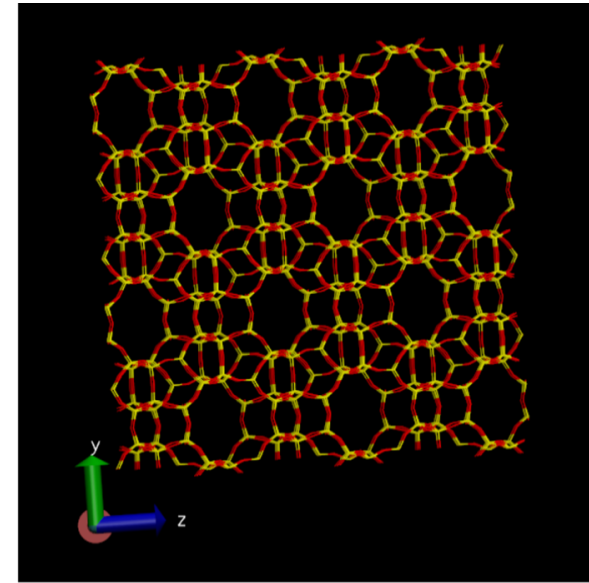

(b)

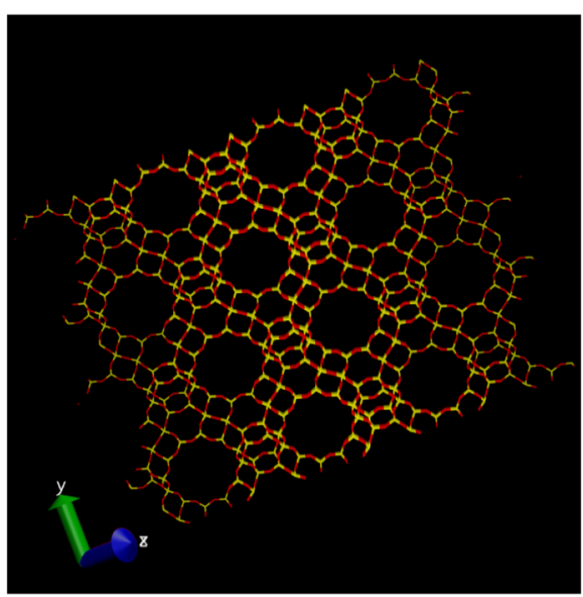

(c)

\section{A. ROZ equations for a two-component matrix}

When building the ROZ equations for Ar adsorbed into a zeolite, one can either neglect the Si atoms (as it is done in the simulation calculations) or include them by means of a purely repulsive interaction. Both cases will be studied, but for the sake of generality, we will introduce the equations for the two component matrix case only. Its reduction to one-component is trivial.

Our model incorporates the zeolite structure solely through the orientationally averaged partial structure factors, which for convenience we define here in their symmetrized form as

$S_{\alpha \beta}(q)=\delta_{\alpha \beta}+\sqrt{\rho_{\alpha} \rho_{\beta}} 4 \pi \int\left(g_{\alpha \beta}(r)-1\right) \frac{\sin (q r)}{q r} r^{2} d r$,

where $g_{\alpha \beta}(r)$ is the partial pair distribution function, $\alpha$ and $\beta$ stand for $\mathrm{Si}$ or $\mathrm{O}$ atoms, $x_{\alpha}$ is the molar fraction, $\rho_{\alpha, \beta}$ are the partial number densities of the $\mathrm{Si}$ and $\mathrm{O}$, and $q$ is the wave vector modulus. These functions contain only pair information, so effects resulting from the fact that $\mathrm{Si}$ atoms are completely screened by $\mathrm{O}$ atoms are partly lost. Moreover, the ordered nature of the porous structure is only contained in the structure factors to a limited extent. We will comment on the effect of these limitations later on.

The explicit procedure for the solution of the ROZ equations can be found in Ref. 29, and we briefly sketch here its main points for completeness.
FIG. 1. Relevant projections of the structure of MEL, MFI, and FAU zeolites. Note that the $x y$ and $x z$ projections of the MEL zeolites are identical, and the $x z$ projection of the MFI zeolite is identical to that of the MEL zeolite. The projection of the FAU zeolite corresponds to the plane 110: (a) MEL, (b) MFI, (c) FAU.
The ROZ equations can be written in matrix form in Fourier space in terms of density scaled Fourier transformed functions as ${ }^{29}$

$$
\begin{aligned}
& \boldsymbol{H}^{01}=\boldsymbol{C}^{01}+\boldsymbol{C}^{00} \boldsymbol{H}^{01}+\boldsymbol{C}^{01} \boldsymbol{H}^{11}-\boldsymbol{C}^{01} \boldsymbol{H}^{12}, \\
& \boldsymbol{H}^{11}=\boldsymbol{C}^{11}+\boldsymbol{C}^{10} \boldsymbol{H}^{01}+\boldsymbol{C}^{11} \boldsymbol{H}^{11}-\boldsymbol{C}^{12} \boldsymbol{H}^{12}, \\
& \boldsymbol{H}^{12}=\boldsymbol{C}^{12}+\boldsymbol{C}^{10} \boldsymbol{H}^{01}+\boldsymbol{C}^{11} \boldsymbol{H}^{12}+\boldsymbol{C}^{12} \boldsymbol{H}^{11}-2 \boldsymbol{C}^{12} \boldsymbol{H}^{12},
\end{aligned}
$$

together with the decoupled equation for matrix-matrix correlations

$$
\boldsymbol{H}^{00}=\boldsymbol{C}^{00}+\boldsymbol{C}^{00} \boldsymbol{H}^{00},
$$

where the superscript 2 denotes the replicas of fluid particles. Now, each of the functions $\boldsymbol{F}^{i j}$ (where $\boldsymbol{F}$ stands for either $\boldsymbol{H}$ or C) can be explicitly expressed in terms of the density scaled Fourier transforms of the total correlation function, $\tilde{h}_{\alpha v}$, or direct correlation function, $\tilde{c}_{\alpha \nu}$, as

$$
\boldsymbol{F}^{01}=\left(\begin{array}{c}
\tilde{f}_{0_{\alpha} 1} \\
\tilde{f}_{0_{\beta} 1}
\end{array}\right), \boldsymbol{F}^{11}=\tilde{f}_{11}, \boldsymbol{F}^{12}=\tilde{f}_{11}^{r},
$$

where the subscripts $\alpha$ and $\beta$ correspond either to Si or O. Correspondingly, for the matrix one has

$$
\boldsymbol{F}^{00}=\left(\begin{array}{cc}
\tilde{f}_{0_{\alpha} 0_{\alpha}} & \tilde{f}_{0_{\alpha} 0_{\beta}} \\
\tilde{f}_{0_{\beta} 0_{\alpha}} & \tilde{f}_{0_{\beta} 0_{\beta}}
\end{array}\right) .
$$

In the equations above, the superscript $r$ denotes correlations between the replicas of the annealed fluid. Additionally, we 
have $\boldsymbol{F}^{10}=\boldsymbol{F}^{01^{T}}$, where the superscript $T$ denotes the matrix transpose.

These equations are complemented by the corresponding closures in $r$-space, which in the HNC approximation read

$$
\begin{aligned}
h_{11}(r) & =\exp \left(-u_{11}(r) / k_{B} T+h_{11}(r)-c_{11}(r)\right)-1, \\
h_{0_{O} 1}(r) & =\exp \left(-u_{0_{O} 1}(r) / k_{B} T+h_{0^{1}}(r)-c_{0_{O} 1}(r)\right)-1, \\
h_{0_{S i} 1}(r) & =\exp \left(-u_{0_{S i} 1}(r) / k_{B} T+h_{0_{S i} 1}(r)-c_{0_{S i} 1}(r)\right)-1, \\
h_{11}^{r}(r) & =\exp \left(h_{11}^{r}(r)-c_{11}^{r}(r)\right)-1,
\end{aligned}
$$

where $f_{0_{i} 1}=f_{10_{i}}$. For the matrix, we would also have

$$
h_{0_{i} 0_{j}}(r)=\exp \left(-u_{0_{i} 0_{j}}(r) / k_{B} T_{0}+h_{0_{i} 0_{j}}(r)-c_{0_{i} 0_{j}}(r)\right)-1 .
$$

Here, $T_{0}$ would be the temperature at which the matrix atoms have been quenched. In our case, we will instead use the "exact" averaged structure factor, given by Eq. (2), which combined with (4) gives

$$
C^{00}=[S-I] S^{-1} .
$$

Here, $\boldsymbol{I}$ is the identity matrix, and the elements of the $\boldsymbol{S}$ matrix are given by Eq. (2). Eq. (9) is then to be inserted into Eq. (3). The latter can be cast into a more compact form

$$
\begin{aligned}
\left(\begin{array}{l}
C^{01} \\
\boldsymbol{C}^{11} \\
\boldsymbol{C}^{12}
\end{array}\right) & =\left(\begin{array}{ccc}
\boldsymbol{I}-\boldsymbol{C}^{00} & -\boldsymbol{C}^{01} & \boldsymbol{C}^{01} \\
-\boldsymbol{C}^{10} & \boldsymbol{I}-\boldsymbol{C}^{11} & \boldsymbol{C}^{12} \\
-\boldsymbol{C}^{10} & -\boldsymbol{C}^{12} & \boldsymbol{I}-\boldsymbol{C}^{11}+2 \boldsymbol{C}^{12}
\end{array}\right) \\
& \times\left(\begin{array}{l}
\boldsymbol{H}^{01} \\
\boldsymbol{H}^{11} \\
\boldsymbol{H}^{12}
\end{array}\right) .
\end{aligned}
$$

Eq. (10) can be efficiently solved for the components of the total correlation function in terms of the direct correlation function using a lower-upper (LU)-decomposition based equation solver. ${ }^{30}$ Eqs. (7) and (10) can now be solved iteratively.

Finally, the adsorption isotherm from the ROZ-HNC can be evaluated computing the chemical potential in terms of the adsorbed fluid density, the former being given by ${ }^{31-33}$

$$
\begin{aligned}
\mu_{1} / k_{B} T= & -\sum_{i=\alpha, \beta} \rho_{0_{i}} \tilde{c}_{0_{i}}(0)-\rho_{1}\left(\tilde{c}_{11}(0)-\tilde{c}_{11}^{r}(0)\right) \\
& +\frac{1}{2} \sum_{i=\alpha, \beta} \rho_{0_{i}} 4 \pi \int d r r^{2} h_{0_{i} 1}(r) \gamma_{0_{i} 1}(r) \\
& +\frac{1}{2} \rho_{1} 4 \pi \int d r r^{2}\left(h_{11}(r) \gamma_{11}(r)\right. \\
& \left.-h_{11}^{r}(r) \gamma_{11}^{r}(r)\right)+\log \left(\rho_{1} \Lambda_{1}^{3}\right),
\end{aligned}
$$

where $\Lambda_{1}$ is the de Broglie wavelength for the fluid particles, and $\gamma(r)=h(r)-c(r)$. For practical purposes and to ease comparison of theory and simulation, we have dropped the temperature dependence of the ideal contribution in (11) and simply set it to $\log \left(\rho_{1} \sigma_{A r A r}^{3}\right)$.

\section{B. The three dimensional Ornstein-Zernike approach}

The inhomogeneous density of a fluid under the influence of the external field created by a set of porous matrix particles can be expressed in terms of a HNC-like expression of the form ${ }^{2,19}$

$$
\begin{aligned}
\rho_{1}(\mathbf{r})= & \bar{\rho}_{1} \\
& \times \exp \left[-U_{01}(\mathbf{r}) / k_{B} T+\int c_{11}\left(\mathbf{r}-\mathbf{r}^{\prime}\right)\left(\rho_{1}\left(\mathbf{r}^{\prime}\right)-\bar{\rho}_{1}\right) d \mathbf{r}\right] .
\end{aligned}
$$

In the context of solvation, $\bar{\rho}_{1}$ is actually the bulk fluid density, and since when $r \rightarrow \infty$ (i.e., well away from the solvated molecule), the homogeneous density must be recovered. As found in Ref. 19, this is no longer the case when the fluid is confined in a porous system, and hence, here $\bar{\rho}_{1}$ will be a parameter to be determined self-consistently. Eq. (12) has the form of Percus source particle approach. ${ }^{34}$ Here, the matrix as a whole is the source of an external potential, $U_{01}(r)$, and therefore, $\rho_{1}(\mathbf{r})=\bar{\rho}_{1} g_{01}(\mathbf{r})$. Additionally, the convolution within the exponential accounts for the matrix fluid indirect correlation function, i.e., matrixfluid correlations mediated by fluid particles. The external potential for a given configuration $\left\{\mathbf{r}_{0}\right\}$ of $N_{0}$ matrix particles reads

$$
U_{0_{\alpha} 1}(x, y, z)=\sum_{i=1}^{N_{0}} u_{0_{\alpha} 1}\left(x-x_{0_{i}}, y-y_{0_{i}}, z-z_{0_{i}}\right) .
$$

When $\alpha=\mathbf{O}, u_{0_{O^{1}}}(r)$ is given by a Lennard-Jones interaction as defined in Section II. Si-Ar interactions are neglected, but in the case of faujasite, auxiliary sites must be included at the center of the isolated cavities. For these sites, $u_{0_{a u x} 1}$ has been defined as a LJ interaction with the same parameters as that of Ar-O but truncated and shifted at $2^{1 / 6} \sigma_{O A r}$, i.e., very short ranged and purely repulsive. This will prevent these cavities from being filled with fluid density when solving the $3 \mathrm{DOZ}$ equation. Now, along the lines of Ref. 19, the fluid-fluid correlations are approximated by the ROZ-HNC, by which $c_{11}\left(\mathbf{r}-\mathbf{r}^{\prime}\right)$ in Eq. (12) is given by

$$
\begin{aligned}
& c_{11}\left(x-x^{\prime}, y-y^{\prime}, z-z^{\prime}\right) \\
& \quad=c_{11}^{\mathrm{ROZ}-\mathrm{HNC}}\left(\left(\left(x-x^{\prime}\right)^{2}+\left(y-y^{\prime}\right)^{2}+\left(z-z^{\prime}\right)^{2}\right)^{1 / 2}\right),
\end{aligned}
$$

and $c_{11}^{\mathrm{ROZ}-\mathrm{HNC}}(r)$ is computed by solution of Eqs. (3) and (7). Once (14) is known, Eq. (12) can be solved iteratively. To that purpose it is conveniently rewritten as

$$
h(x, y, z)=\exp \left[-U_{01}(x, y, z) / k_{B} T+\bar{\rho}_{1} \int d x^{\prime} d y^{\prime} d z^{\prime} c_{11}\left(x-x^{\prime}, y-y^{\prime}, z-z^{\prime}\right) h\left(x^{\prime}, y^{\prime}, z^{\prime}\right)\right]-1,
$$

and

$$
\rho_{1}(x, y, z)=\bar{\rho}_{1}(h(x, y, z)+1) .
$$




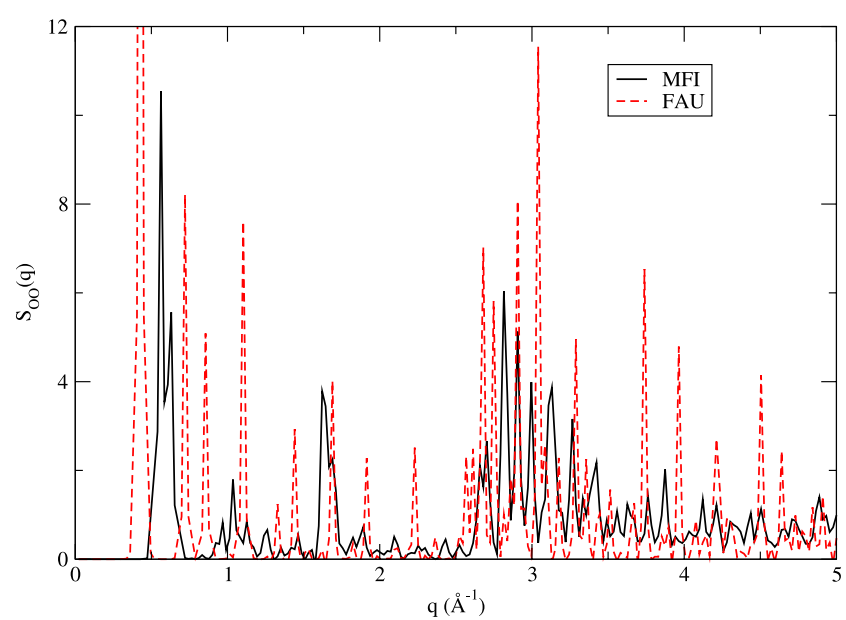

FIG. 2. Oxygen-oxygen structure factors calculated for MFI and FAU zeolites as defined in Eq. (2).

The convolution in (15) is evaluated in Fourier space using numerical Fourier transforms from the FFTW3 library. ${ }^{35}$ Our problem is intrinsically periodic (as long as fluid correlations are of shorter range than the zeolite super-cell being used). For that reason, the Fourier transforms can be carried out without zero padding.

As mentioned before, in Eq. (15), the value of the average density $\bar{\rho}_{1}$ must be defined self-consistently. To that aim, we

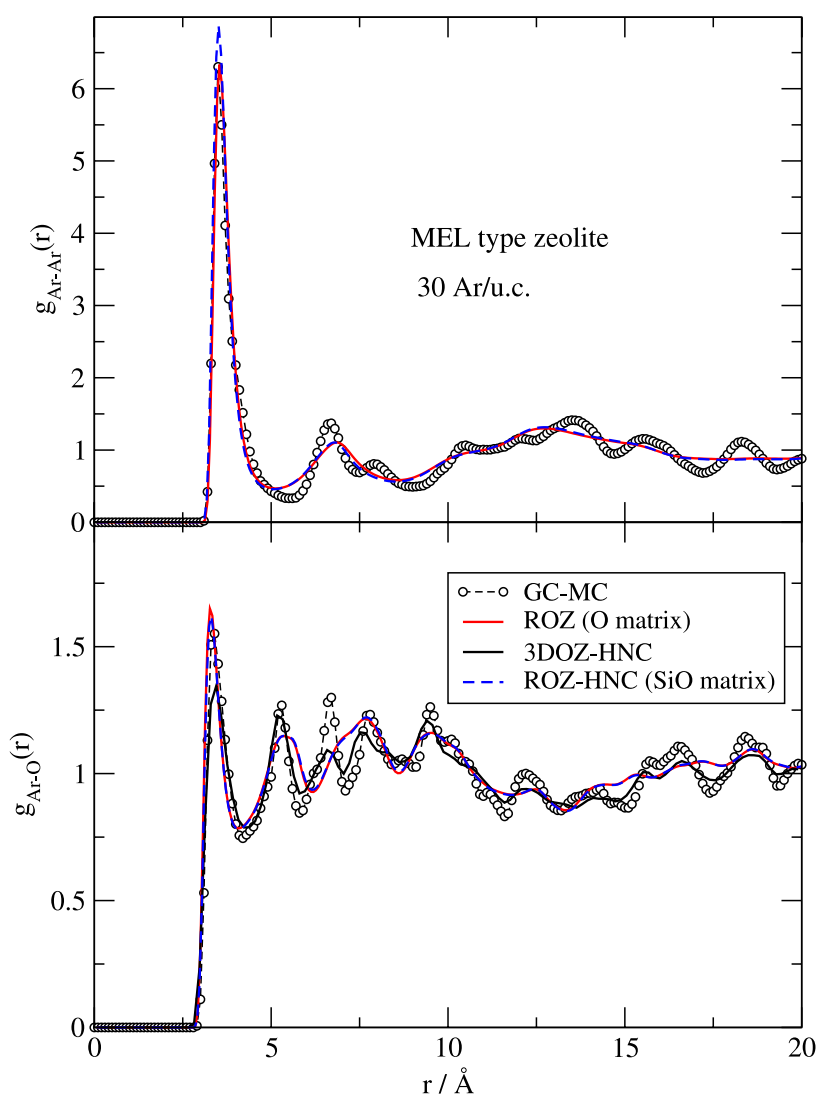

FIG. 3. Fluid-fluid and zeolite-fluid average pair distribution functions for Ar adsorbed into a MEL-zeolite at $77 \mathrm{~K}$. Simulation results are denoted by circles, red curves represent results from the ROZ-HNC equations with a onecomponent matrix, dashed-dotted blue curves those of the two component matrix, and solid black curve corresponds to results from the 3DOZ-HNC at $94 \mathrm{~K}$. simply resort to the sum rule of the density distribution ${ }^{19}$

$$
\left\langle\rho_{1}\right\rangle=\frac{\bar{\rho}_{1}}{L_{x} L_{y} L_{z}} \int d x d y d z(h(x, y, z)+1),
$$

where $L_{x}, L_{y}, L_{z}$ are the dimensions of the periodic cell, and the average fluid density is defined as

$$
\left\langle\rho_{1}\right\rangle=\frac{N_{1}}{L_{x} L_{y} L_{z}} .
$$

Here, $N_{1}$ is the number of adsorbate atoms (Ar in our case) confined in the zeolite super-cell. In this way, given an average fluid density, $\left\langle\rho_{1}\right\rangle$, the parameter $\bar{\rho}_{1}$ can be evaluated iteratively solving Equations (15) and (17) self-consistently, i.e., modifying $\bar{\rho}_{1}$ until the desired average fluid density $\left\langle\rho_{1}\right\rangle$ is achieved. Recall that the ROZ-HNC equations must be solved in advance for the averaged fluid density, $\left\langle\rho_{1}\right\rangle$, and the corresponding density of the confining medium, $\rho_{0}$. These data specify the problem and are known in advance from experimental techniques.

Finally, once the fluid spatial density distribution, $\rho_{1}(x, y$, $z$ ), is found, the matrix-fluid correlations averaged over the inhomogeneities can also be evaluated. One simply calculates the average over matrix positions

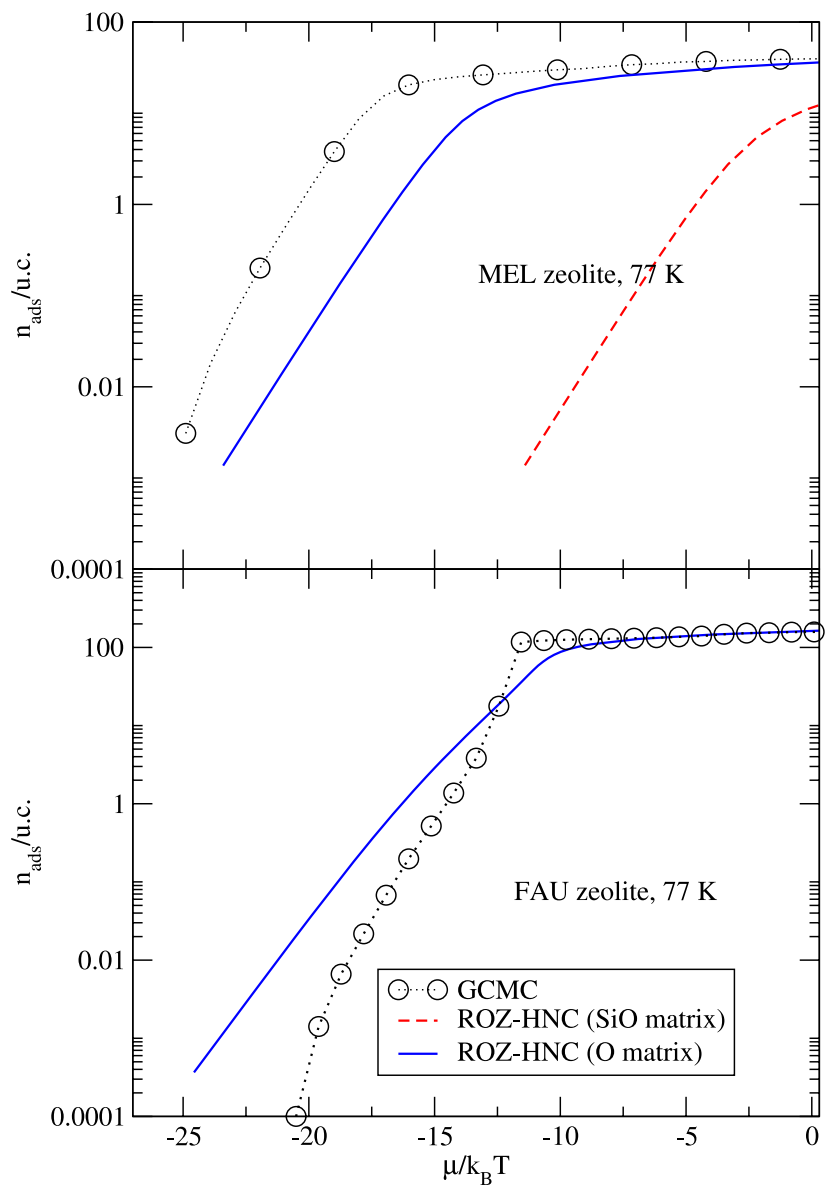

FIG. 4. Adsorption isotherms of Ar in MEL (upper graph) and FAU zeolites (lower graph) obtained from GCMC simulations and from the ROZ-HNC approximation with one and two component matrices as indicated in the legend. 
$g_{01}(r)=\frac{1}{\bar{\rho}_{1} N_{0}} \sum_{i=1}^{N_{0}} \int \rho_{1}\left(\mathbf{r}^{\prime}-\mathbf{r}_{0_{i}}\right) \delta\left(\mathbf{r}-\mathbf{r}^{\prime}+\mathbf{r}_{0}\right) d \mathbf{r}^{\prime}$,

where the integration over the Dirac's $\delta\left(\mathbf{r}-\mathbf{r}^{\prime}+\mathbf{r}_{0}\right)$ can be discretized as

$$
\begin{aligned}
g_{01}(r)= & \frac{1}{\bar{\rho}_{1} N_{0}} \sum_{m=1}^{N_{0}} \sum_{i, j, k} \rho_{1}\left(x_{i}^{\prime}, y_{j}^{\prime}, z_{k}^{\prime}\right) H\left(r+\Delta r-r_{m}^{\prime}\right) \\
& \times H\left(r_{m}^{\prime}-r+\Delta r\right) \frac{\Delta x \Delta y \Delta z}{\Delta V_{\text {shell }}} .
\end{aligned}
$$

In this equation, $r_{m}^{\prime}=\sqrt{\left(x_{i}^{\prime}-x_{m}\right)^{2}+\left(y_{j}^{\prime}-y_{m}\right)^{2}+\left(z_{k}^{\prime}-z_{m}\right)^{2}}$, $H(x)$ is a step function, and the space is discretized according to $x_{i}^{\prime}=i \Delta x, y_{j}^{\prime}=j \Delta y$ and $z_{k}^{\prime}=k \Delta z . \Delta V_{\text {shell }}$ defines the volume of the spherical shell (circular in two dimensional problems) determined by the discretized Dirac $\delta$, i.e., here $\Delta V_{\text {shell }}=4 \pi r^{2} \Delta r$. For practical purposes, we have chosen our grid in $r$ such that $\Delta r=\Delta x$. The summation in (19) and (20) runs over the number of matrix particles, or alternatively a representative number of them, e.g., over the unit cell in a periodic system. We note in passing that expression (12) in Ref. 19, although numerically correct in 2D, is not general and it is inappropriate in three dimensions.

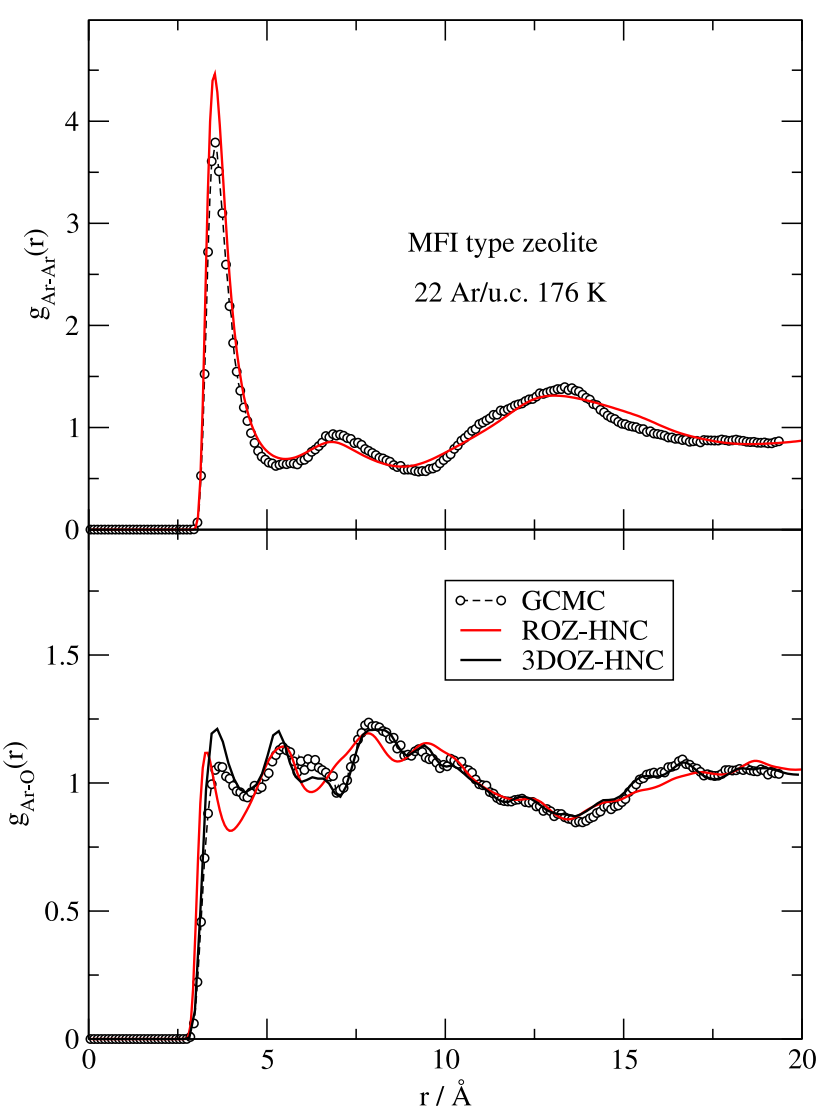

FIG. 5. Fluid-fluid and zeolite-fluid average pair distribution functions for Ar adsorbed into a MFI-zeolite at $176 \mathrm{~K}$. Simulation results are denoted by circles, red curves represent results from the ROZ-HNC equations (onecomponent matrix), and solid black curve corresponds to results from the 3DOZ-HNC.

\section{RESULTS}

Of the three zeolite frameworks considered in this work, MFI and MEL have a very similar structure, and Ar atoms adsorbed into them are under conditions of very tight confinement. In both instances, the channel width can only accommodate one atom, and the channel portion between intersections two atoms. Channel intersections have room for up to 4-6 atoms. As a whole, these rigid zeolite models can accommodate 30-40 atoms per unit cell. In contrast, FAU frameworks accept a loading of up to $150 \mathrm{Ar}$ atoms per unit cell. These different levels of confinement will have a significant reflection in the performance of the theoretical approach. In order to assess the quality of our approximations, we have performed GCMC simulations for the adsorption isotherms of Ar in the three zeolite frameworks at $77 \mathrm{~K}$ for MEL and FAU and at $176 \mathrm{~K}$ for MFI. These simulations were $2 \times 10^{6}$ steps long, of which the first 500000 were used for equilibration. Each step implies a displacement attempt of all the sample particles, one deletion attempt and one insertion attempt. The procedure uses a cavity bias algorithm to speed up the simulation. ${ }^{36}$ Three dimensional density maps of the adsorbed fluid were then generated for MEL at $77 \mathrm{~K}$ and a load of $30 \mathrm{Ar}$ atoms/u.c., for MFI at $176 \mathrm{~K}$ and a load of $22 \mathrm{Ar}$ /u.c., and for FAU for $104 \mathrm{Ar} /$ u.c. at $77 \mathrm{~K}$. For that purpose from the final GCMC configurations for a given load, canonical Molecular Dynamics (MD) simulations were run for $5 \times 10^{6}$ steps and averages were calculated over 5000 configurations separated by 1000

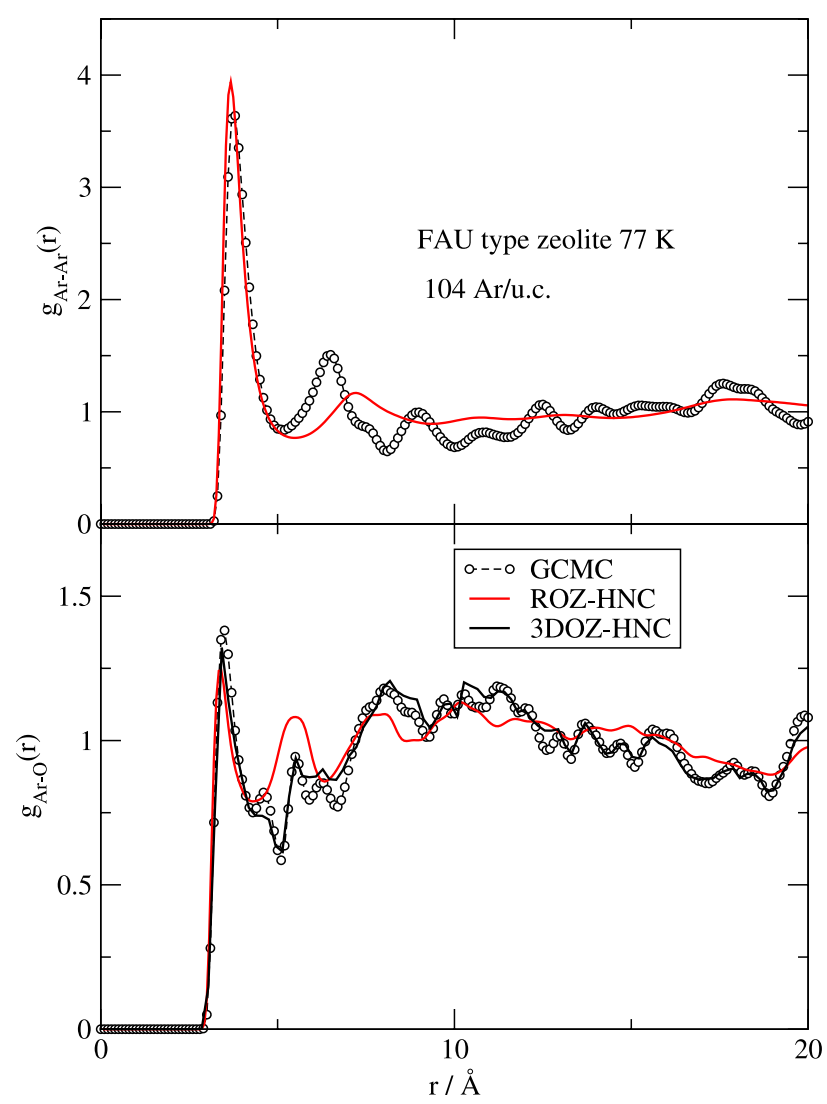

FIG. 6. Fluid-fluid and zeolite-fluid average pair distribution functions for $\mathrm{Ar}$ adsorbed into a FAU-zeolite at $77 \mathrm{~K}$. Simulation results are denoted by circles, red curves represent results from the ROZ-HNC equations (one-component matrix), and solid black curve corresponds to results from the 3DOZ-HNC. 
steps. The loads were chosen such that in the case of MEL is close to the saturation and above the adsorption step present in the experimental isotherm, ${ }^{37}$ and for MFI is just below the load corresponding to the adsorption step found at $77 \mathrm{~K} .{ }^{25}$ In the case of FAU, the loading corresponds to two thirds of saturation, and the adsorption isotherm does not present now any singular feature.

As a first step to solve the 3DOZ-HNC equation, we first have to solve the ROZ-HNC equations. This we have done on a 2048 point grid with a grid size of $0.02 \sigma_{A r A r}$ using standard procedures. ${ }^{29}$ As mentioned, as input data our calculations require the knowledge of the structure factor. Since the zeolite structures are well-known, the structure factors can be accurately determined. However, the periodic nature of the structure prevents the use of Eq. (2), and one has to resort to a direct $q$-sampling alternative. This can be done using standard procedures, e.g., Eq. (1) in Ref. 38, and as an illustration, results for FAU and MFI are plotted in Figure 2. With these structure factors, once symmetrized as in Eq. (2), one can solve the ROZ equation, both using either a single component $(\mathrm{O})$ matrix or a two component $(\mathrm{Si} / \mathrm{O})$ matrix. What we have found is that the structural results of the two component matrix are practically indistinguishable from those of the single component matrix (see Figure 3 where the theoretical Ar-Ar and Ar$\mathrm{O}$ pair distribution functions are compared with simulation results). In contrast, the adsorption isotherms of the ROZ-HNC using the two component matrix are considerably worse (see Figure 4 for MEL). The problem clearly stems from the fact that including the $\mathrm{Si}$ atoms in the ROZ calculation increases the matrix density (i.e., lowers the porosity), and the atom-atom

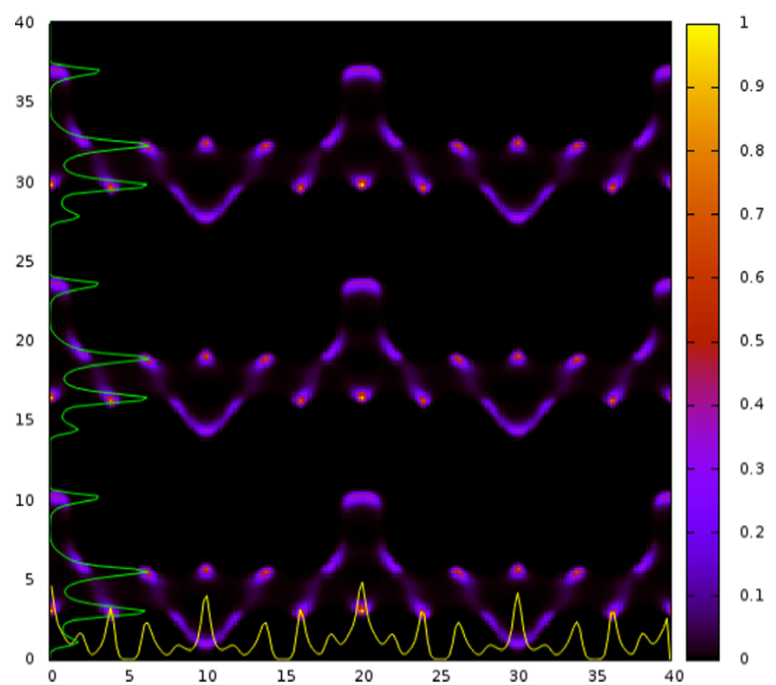

(a)

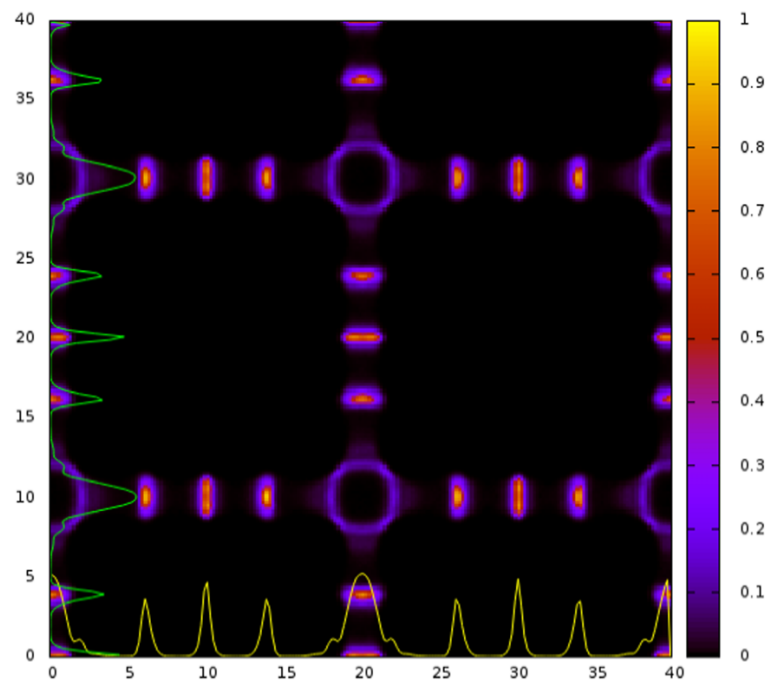

(c)

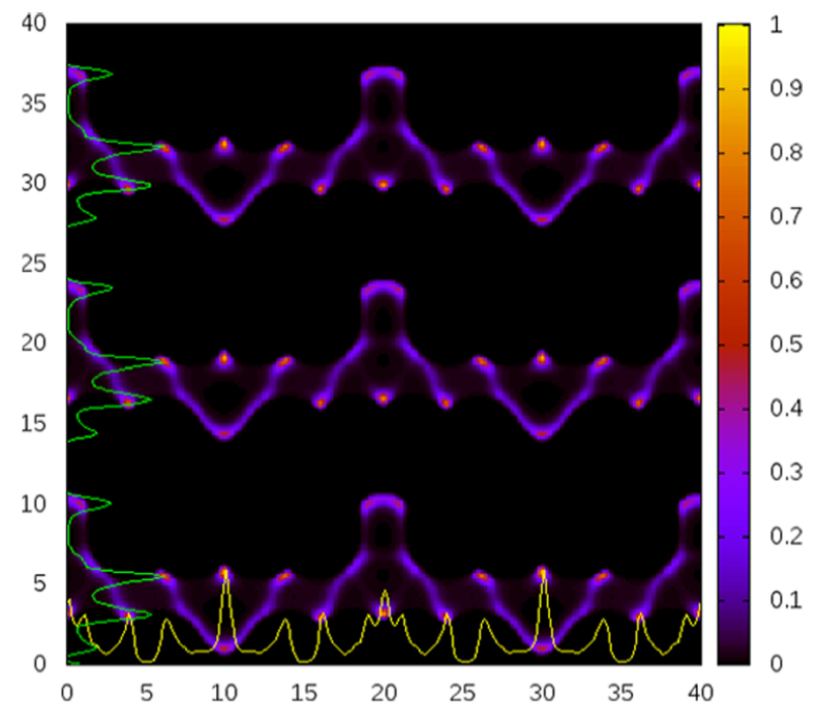

(b)

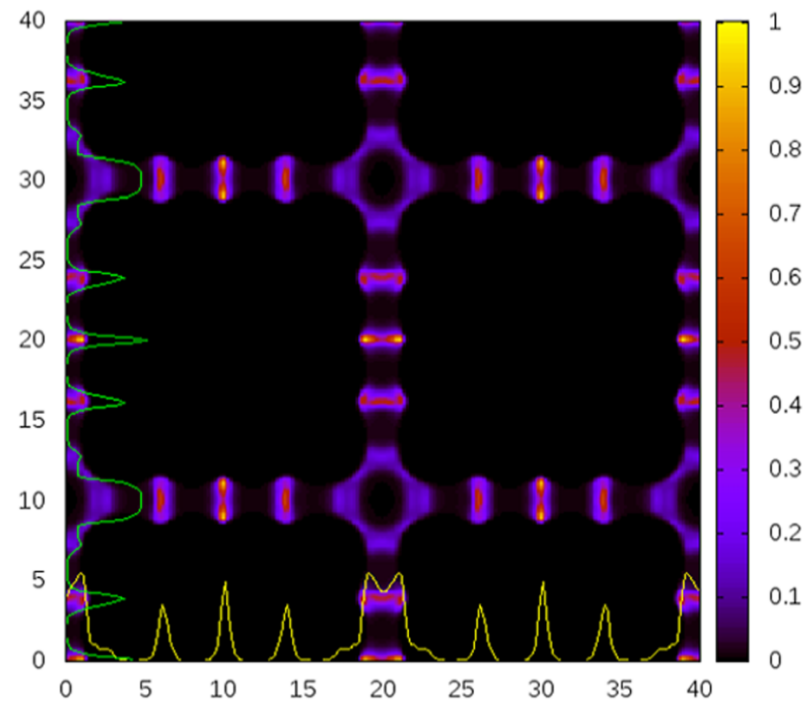

(d)

FIG. 7. Scaled fluid density distribution $\rho_{1}(x, y, z) / \rho^{\max }$ of adsorbed Ar atoms into a MEL zeolite corresponding to a $1.5 \AA$ thick slab centered at $y_{0}=20.36 \AA$ for the $x z$-plane and for the $x y$-plane at $z_{0}=23.4 \AA$ as computed from the 3DOZ-HNC equation for $T=94 \mathrm{~K}$ and $32 \mathrm{Ar} / \mathrm{u} . c$. and simulation results for the same load and $77 \mathrm{~K}$. Density profile projections on the $y$-and $z$-axis for a $1.5 \AA$ thick slab are illustrated by green and yellow curves arbitrarily normalized to ease the visibility of the graphs. The plane positions are chosen to illustrate the channel structure. $\rho^{\max }$ is chosen as the maximum value of $\rho_{1}(x, y, z)$ - both in the $3 \mathrm{DOZ}$ and MD results — so as to normalize the color scale of the plot. (a) $\mathrm{MD} \rho_{1}\left(x, y_{0}, z\right) / \rho^{\max }$, (b) $3 \mathrm{DOZ} \rho_{1}\left(x, y_{0}, z\right) / \rho^{\max }$, (c) MD $\rho_{1}\left(x, y, z_{0}\right) / \rho^{\max }$, (d) $3 \mathrm{DOZ} \rho_{1}\left(x, y, z_{0}\right) / \rho^{\max }$. 
structure factors contain only partial information on the zeolite structure. The fact that the $\mathrm{Si}$ atoms are embedded in the $\mathrm{O}$ tetrahedra and do not decrease porosity cannot be reliably captured by pairwise structural functions. The net result is an adsorption isotherm that corresponds to a material with a substantially lower porosity.

Consequently, when the two component matrix is used in ROZ equations, the adsorption isotherm is very poorly predicted, as can be seen in Figure 4. If we now focus on the adsorption results for the one component matrix, we observe that both in the case of MEL and FAU zeolites the saturation behavior is correctly reproduced. This is somehow telling us that the knowledge of the matrix density (that of the $\mathrm{O}$ atoms that form the channel structure) and the corresponding structure factors is sufficient to account for the volume available to the adsorbate. This is the quantity that determines the maximum load. Interestingly, the quality of the ROZ results deteriorates at low loads, where adsorption is mostly determined by energetic factors. Here, we observe that the adsorption in MEL zeolites is underestimated and in FAU is overestimated. This low adsorption load regime basically follows Henry's law, i.e., $n_{a d s}=K_{H} \exp \left(\mu / k_{B} T\right)$. The theory

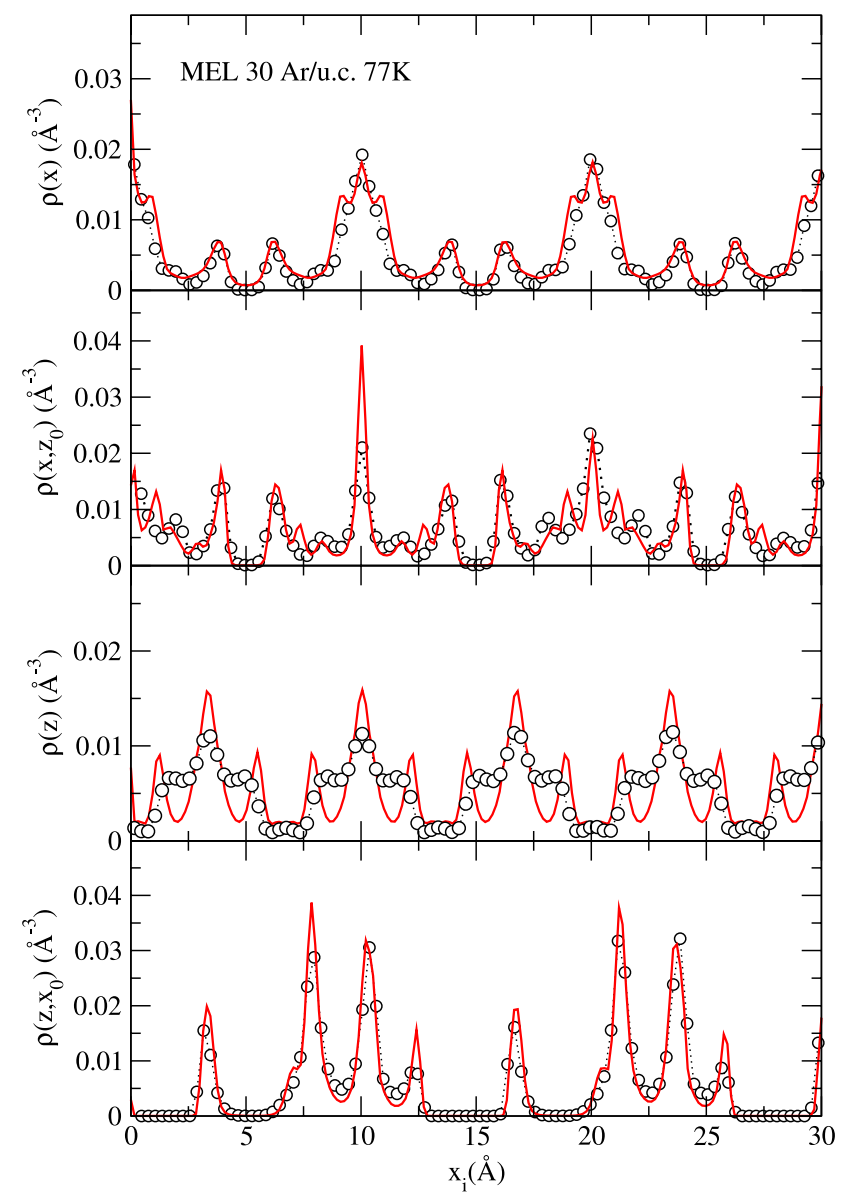

FIG. 8. Cumulative average density profiles of Ar atoms projected on the $x$ axis, $\rho(x)$, and the $z$ axis, $\rho(z)$, and sections $\rho\left(x, z_{0}\right)$ and $\rho\left(z, x_{0}\right)$ for slabs of $1.5 \AA$ thickness around $x_{0}=20.36 \AA$ and $z_{0}=23.4 \AA$ for a load of $30 \mathrm{Ar}$ atoms adsorbed into a MEL zeolite at $77 \mathrm{~K}$, obtained from the 3DOZ-HNC approximation (solid curve) and computer simulation (circles). Note that the 3DOZ-HNC results correspond to the lowest converging temperature, $94 \mathrm{~K}$. The axis label $x_{i}$ denotes $x$ or $z$ depending on the graph under consideration. predicts basically the same adsorption in MEL and in FAU at low chemical potentials $\left(\mathrm{K}_{H}(\mathrm{MEL})=0.0125\right.$ atoms $/$ u.c. vs $\mathrm{K}_{H}(\mathrm{FAU})=0.0114$ atoms/u.c. $)$. This is not what happens in the simulation. When the number of adsorbate atoms is low, MEL adsorbs substantially more than FAU at the same chemical potential as a result of the stronger interactions between Ar atoms and the narrower channels of the MEL. In this case, an adsorbate molecule interacts directly with all the surrounding $\mathrm{O}$ atoms forming the channel, whereas in the case of FAU, the Ar-O interaction takes place mostly with part of channel surface, given the much larger size of the channels. Obviously in the case of low load, Ar-Ar contributions to the net interaction are negligible. It is clear that this difference is strongly dependent on the topology of the channel network, and this is only partially incorporated into the ROZ equations via the structure factors. Since these are orientationally averaged pair functions that enter into a set of equations where all the functions are assumed to be homogeneous, one can hardly expect that such effects could be reproduced by the theory. In fact the latter behaves as if the pore network of its averaged "quenched" matrix would be somewhere in between that of MEL (narrow pores) and FAU (wide pores) zeolites.

Now, in Figures 5 and 6, we present the corresponding pair distribution functions for MFI and FAU zeolites at a somewhat lower relative loading. In these instances, only the single component matrix ROZ results are plotted. We note in passing that at the pair level structure, the ROZ results for the MEL and MFI are practically indistinguishable (and the simulation

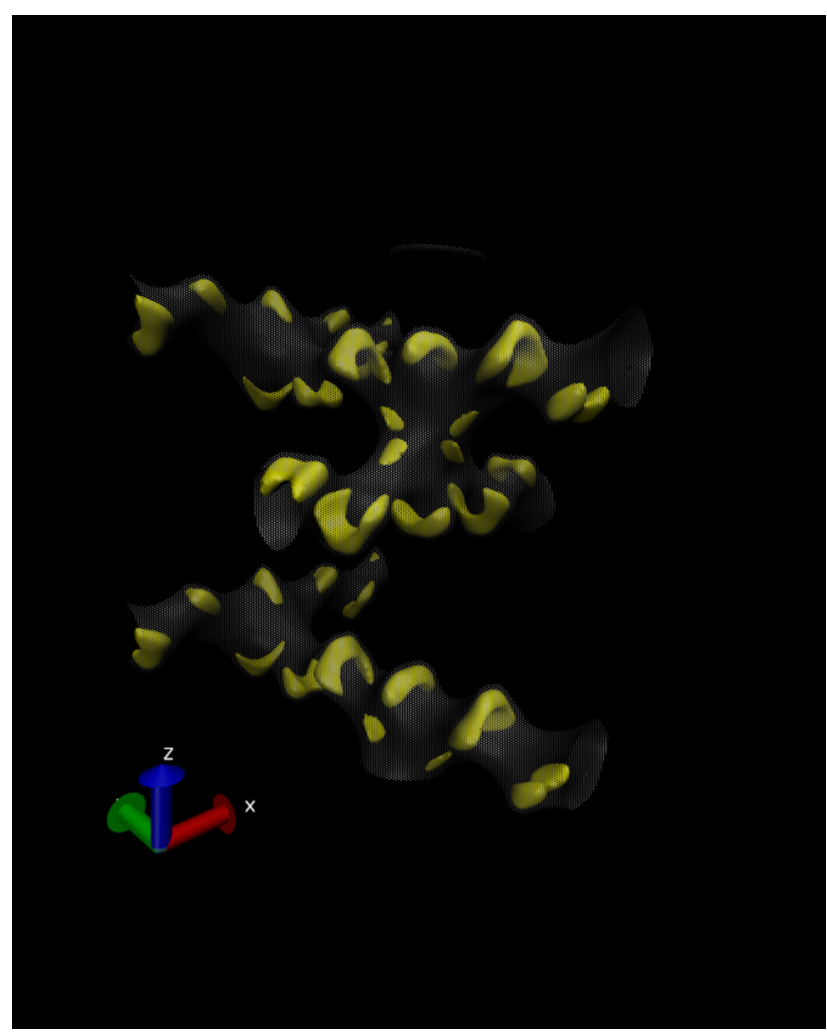

FIG. 9. Three-dimensional density distribution of Ar atoms for a load of 30 Ar atoms adsorbed into a MEL zeolite at $94 \mathrm{~K}$ obtained from the 3DOZHNC approximation, plotted by means of isosurfaces defined by $\rho_{1}(x, y, z)$ $=0.005 \AA^{-3}$ (glassy surface) and $0.058 \AA^{-3}$ (yellow). 
results for the pair distribution functions averaged over the inhomogeneity are also extremely close). In any case, when analyzing the results of Figures 3, 5, and 6, it is rather obvious that the crude ROZ approximation we are using provides a qualitatively correct picture of the pair distribution functions averaged over the lattice inhomogeneities. Note however that the results for FAU (in particular for $g_{A r A r}$ ) are considerably worse than those for MFI or MEL. One might speculate that this can be related to the fact that $\mathrm{Ar}$ in the much larger cavities and channels of FAU is probably better represented by a fluid confined in a cylindrical cavity than in a disordered array of matrix particles. In the case of MEL/MFI, the higher degree of confinement can be somewhat better described from the structural point of view by our disordered matrix model.

We are now in a position to solve the 3D-HNC for our systems of interest. This we have done on an $N \times N \times N$ grid $(N$
$=256$ ), with a grid step defined in terms of the zeolite supercell under consideration, i.e., $\Delta x=L_{x} / N, \Delta y=L_{y} / N, \Delta z$ $=L_{z} / N$, and the cell dimensions $L_{x} \times L_{y} \times L_{z}$ set to $41.14 \AA$ $\times 39.84 \AA \times 40.26 \AA$ for MFI, 40.12 $\mathrm{A} \times 40.12 \AA \times 40.209 \AA$ for MEL, and 48.69 $\AA 48.69 \AA \times 48.69 \AA$ for FAU. Solving the 3DOZ-HNC with these conditions takes approximately $800 \mathrm{Mb}$ of RAM and two minutes on an Intel I7 processor at $3.40 \mathrm{GHz}$ using an Nvidia GPU GeForce $\mathbb{R}$ GTX ${ }^{\mathrm{TM}}$ 590 to evaluate the interaction potential grid and Eq. (20). Using a $512^{3}$ grid increases the computing time up to 13 min and implies an eighth-fold increase in memory usage. In our case, no significant change in the results was appreciated.

When solving the 3DOZ-HNC for MEL and MFI zeolites, we could not find convergence below $94 \mathrm{~K}$. This convergence difficulties are most likely associated with the extremely negative values of the zeolite-Ar interaction in the exponential

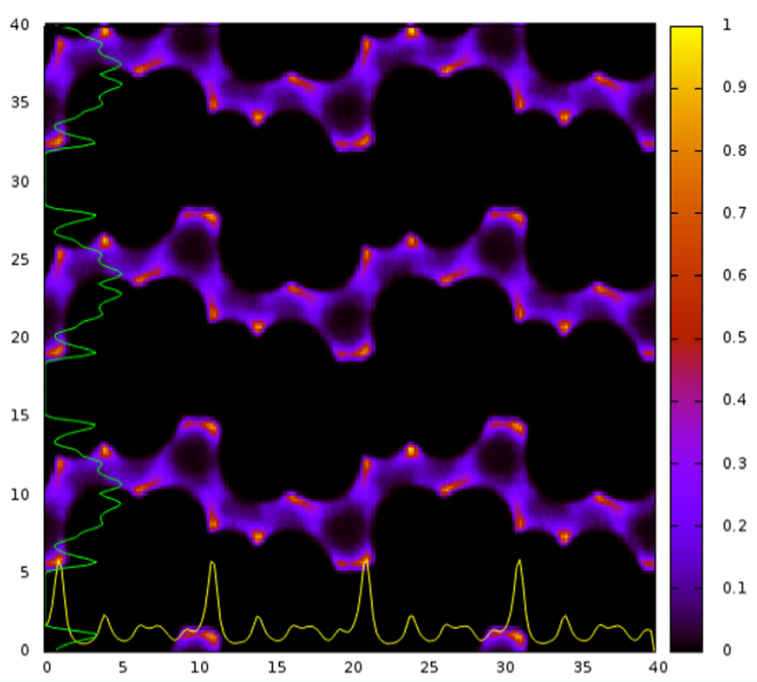

(a)

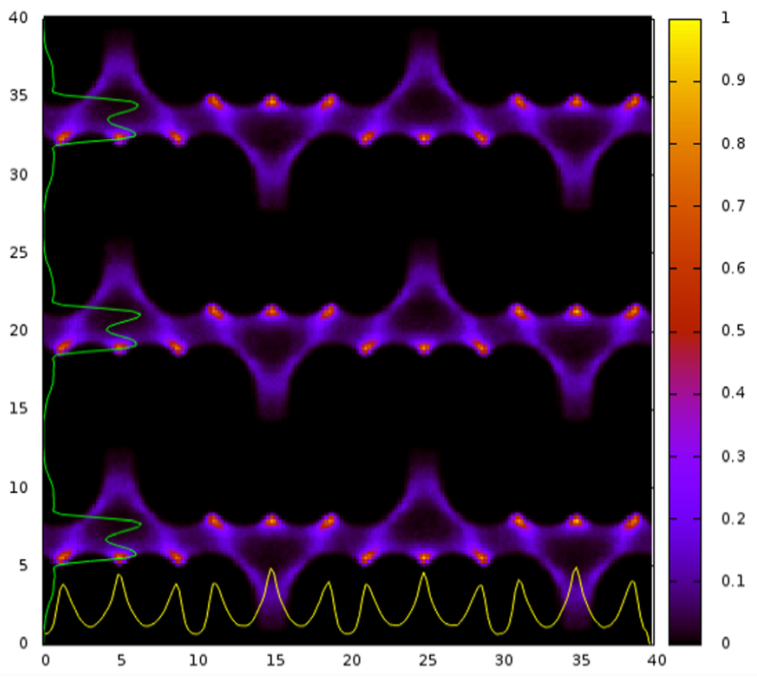

(c)

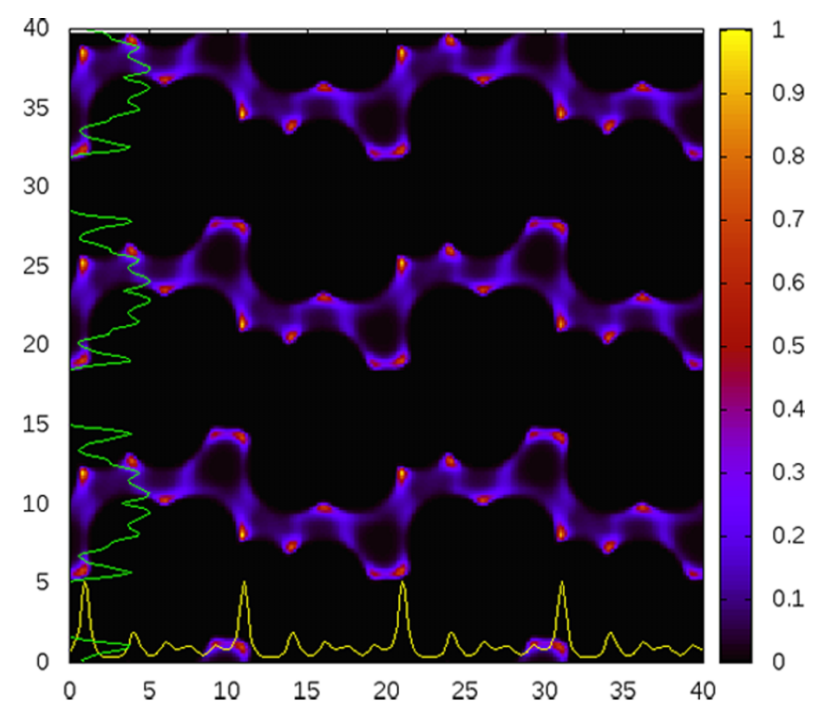

(b)

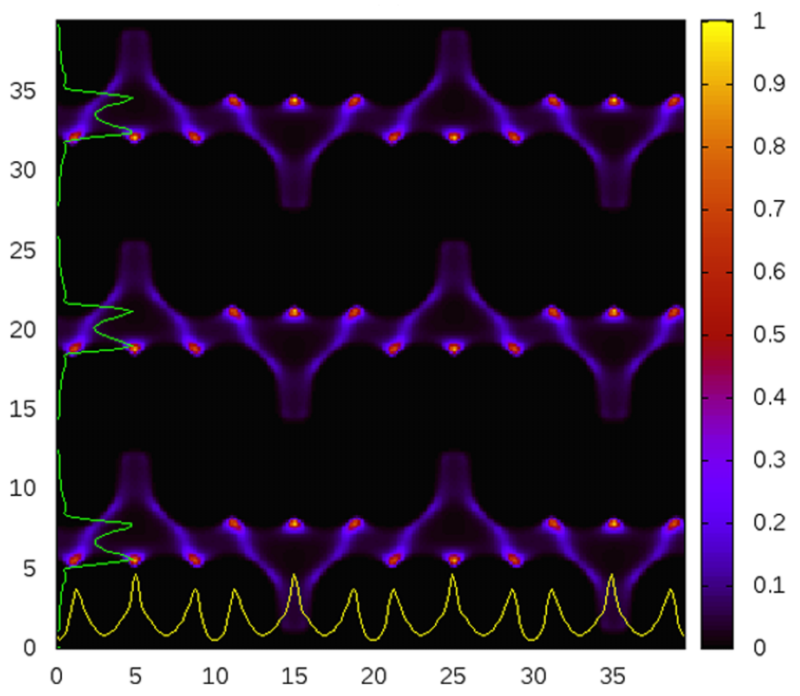

(d)

FIG. 10. Scaled fluid density distribution $\rho_{1}(x, y, z) / \rho^{\max }$ of adsorbed Ar atoms into a MFI zeolite corresponding to a $1.5 \AA$ thick slab placed at $x_{0}=20.04 \AA$ for the $y z$-plane and for the $x z$-plane at $y_{0}=20.36 \AA$ as computed from the 3DOZ-HNC equation and MD simulations for $T=176 \mathrm{~K}$ and $22 \mathrm{Ar} / \mathrm{u}$.c. Density profile projections on the $y$-and $z$-axis for a $1.5 \AA$ thick slab are illustrated by green and yellow curves arbitrarily normalized to ease the visibility of the graphs. The plane positions are chosen to illustrate the channel structure. $\rho^{\max }$ is chosen as the maximum value of $\rho_{1}(x, y, z)$ - both in the 3DOZ and MD results so as to normalize the color scale of the plot. (a) $\operatorname{MD} \rho_{1}\left(x_{0}, y, z\right) / \rho^{\max }$, (b) $3 \mathrm{DOZ} \rho_{1}\left(x_{0}, y, z\right) / \rho^{\max }$, (c) $\operatorname{MD} \rho_{1}\left(x, y_{0}, z\right) / \rho^{\max }$, (d) $3 \mathrm{DOZ} \rho_{1}\left(x, y_{0}, z\right) / \rho^{\max }$. 
of Eq. (15). These difficulties have also been encountered in solvation problems, ${ }^{12}$ and as a possible solution, we intend to investigate the performance of alternative integral equation closures. In any case, in Figures 7 and 8 we present our results for the most unfavorable situation, in which the desired temperature could no be reached. We insist on comparing theory and simulation in these conditions even when temperatures are rather different, since $77 \mathrm{~K}$ is one the reference temperatures for adsorption experiments of noble gases. In these figures, one can see the projection of the fluid density on some relevant planes of the zeolite structure that reflect the channel filling. Note that the MD results are averaged over a slab of $1.5 \AA$ of thickness in order to collect sufficient statistics during the simulation run. Obviously, the theory can provide results with a resolution of approximately $0.2 \AA$ (depending on the grid size) but we have performed a corresponding average over $1.5 \AA$ to be able to compare with the simulation. One immediately appreciates that the theory reproduces qualitatively all the details of the spatial distribution within the channels. This can be seen from a more quantitative perspective in Figure 8, where the density profiles are plotted. One observes that both $\rho(x)$ and $\rho(y)$ are more structured in the theoretical results. This discrepancy is

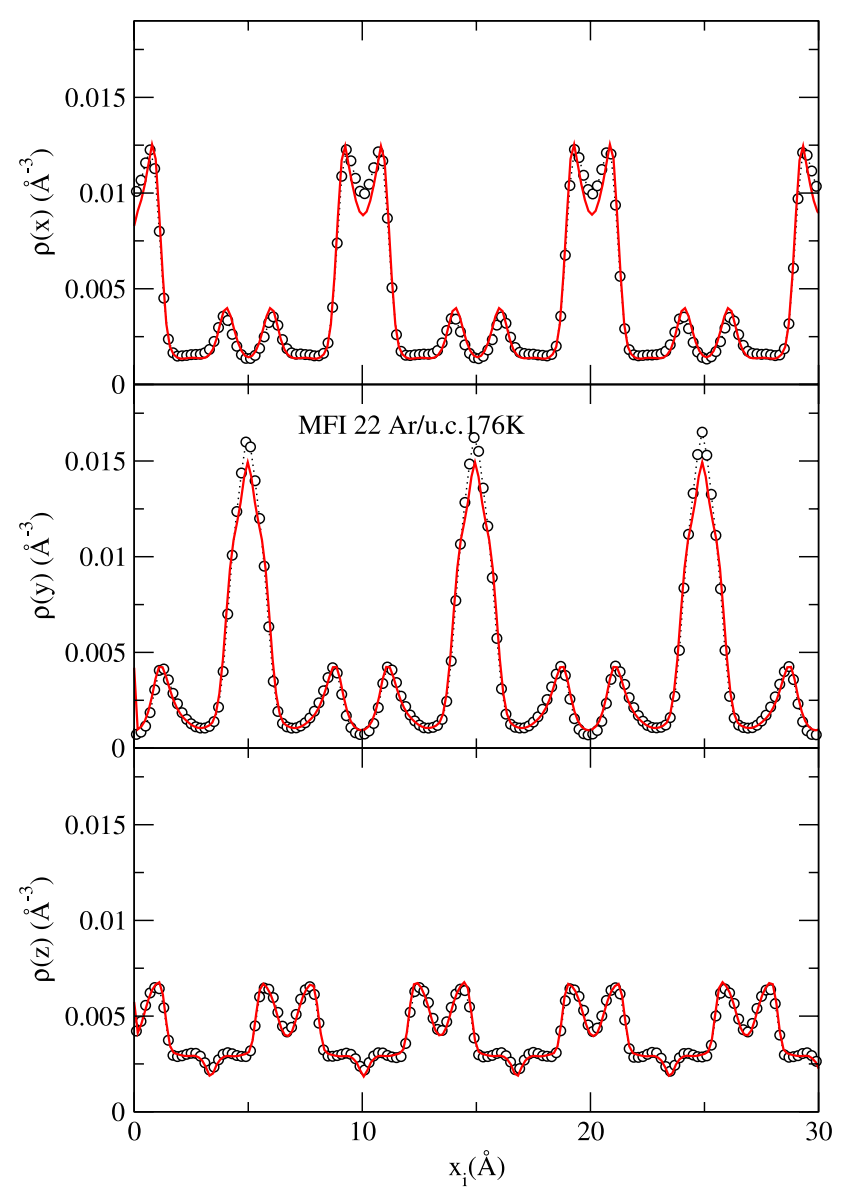

FIG. 11. Cumulative average density profile of $\mathrm{Ar}$ atoms projected on the $x$ axis, $\rho(x), y$-axis, $\rho(y)$, and $z$ axis, $\rho(z)$ for a load of 22 Ar atoms adsorbed into a MFI zeolite at $176 \mathrm{~K}$ obtained from the 3DOZ-HNC approximation (solid curve) and computer simulation (circles). The axis label $x_{i}$ denotes $x$, $y$, or $z$ depending on the graph under consideration. most likely due to the characteristic enhanced correlations of the HNC closure at contact, that in our case might as well be responsible for the breakdown of the equation when lowering the temperature. Additionally, in the lower graph of Figure 3 we have plotted the estimate of $g_{A r O}$ obtained using Eq. (20). Here, one appreciates that the first peak is underestimated, which is a consequence of the inability to reach the correct temperature. Aside from that, the equation reproduces the detailed structure of the distribution function which is somewhat smeared out by the ROZ-HNC (e.g., the third maximum). In Figures 5 and 6, one can more clearly appreciate that the use of Eq. (20) leads to even more accurate results in the case of the MFI at somewhat lower loads and higher temperature, and for FAU at high load/low temperature. Again in the case of MFI, there is a slight overestimation of the peak heights, but for FAU the agreement is almost perfect. This feature is a likely consequence of the lower degree of confinement in this latter instance and subsequent less intense Ar-zeolite net interaction. The considerable improvement over the ROZ-HNC results stems from the fact that the 3DOZ-HNC incorporates the explicit three dimensional description of the confining medium through the potential

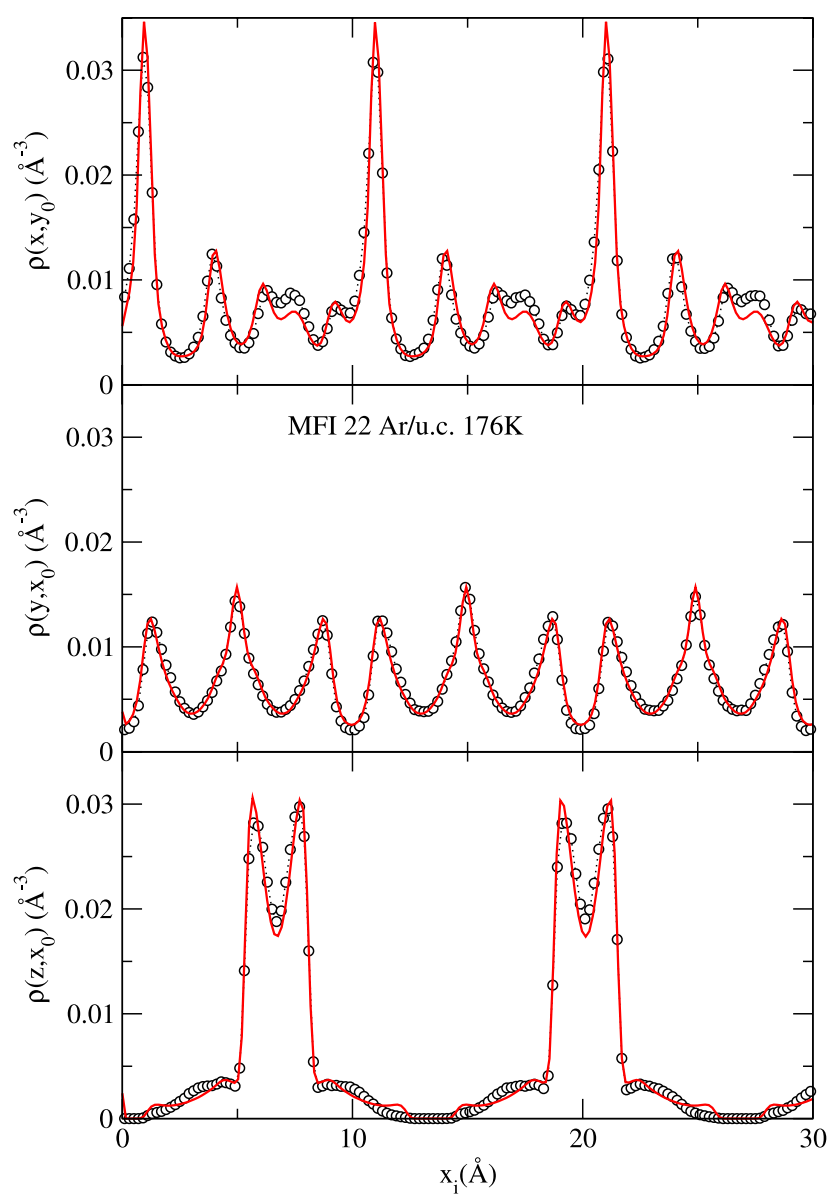

FIG. 12. Sections of the density profiles of Ar atoms projected on the $x$ axis, $\rho\left(x, y_{0}\right)$ for $y_{0}=24.90 \AA, y$-axis, $\rho\left(y, x_{0}\right)$ for $x_{0}=20.04 \AA$, and $z$-axis planes, $\rho\left(z, x_{0}\right)$ for $x_{0}=20.04 \AA$ for a slab of $1.5 \AA$ thickness around the planes for a load of $22 \mathrm{Ar}$ atoms adsorbed into a MFI zeolite at $176 \mathrm{~K}$ obtained from the 3DOZ-HNC approximation (solid curve) and computer simulation (circles). The axis label $x_{i}$ denotes $x, y$, or $z$ depending on the graph under consideration. 
$U_{01}(x, y, z)$. These many body effects are obviously missing in a pairwise approach such as the ROZ-HNC equation, and this explains its somewhat poorer performance in this context.

In summary, despite the limitations due to the convergence difficulties, the 3DOZ-HNC approach seems to provide an accurate description of the spatial distribution of the adsorbed fluid under conditions of strong confinement. As an additional piece of information, in Figure 9 we have plotted a three dimensional representation of the fluid density, represented by means of isosurfaces. A first isosurface represented in transparent glass corresponds to $\rho_{1}(x, y, z)=0.005 \AA^{-3}$, which is close to the average fluid density within the zeolite. In yellow, we indicate areas of high fluid concentration (close to liquid density) $\rho_{1}(x, y, z)=0.058 \AA^{-3}$. One can appreciate the presence of a high density region within the channels and two wide regions on the bottom and the top of the intersections together with 4 small regions in the middle of the intersection. These correspond to the locations where the $6 \mathrm{Ar}$ atoms will be found at full loading. ${ }^{37}$ This figure is a clear illustration of the information on the spatial disposition of the adsorbates that this type of approach can furnish.

In Figure 10, we present density maps for the MFI zeolite under less harsh conditions (a load of $22 \mathrm{Ar}$ atoms/u.c. and $176 \mathrm{~K})$. Results of the integral equation are now fully converged at the desired average adsorbate density and temperature. One observes that the agreement is now almost perfect. This is even more clearly seen in Figure 11 where the cumulative density profiles on the $x$ and $y$ axes are represented, and in Figure 12 where we plot sections of the profiles for slabs around specific

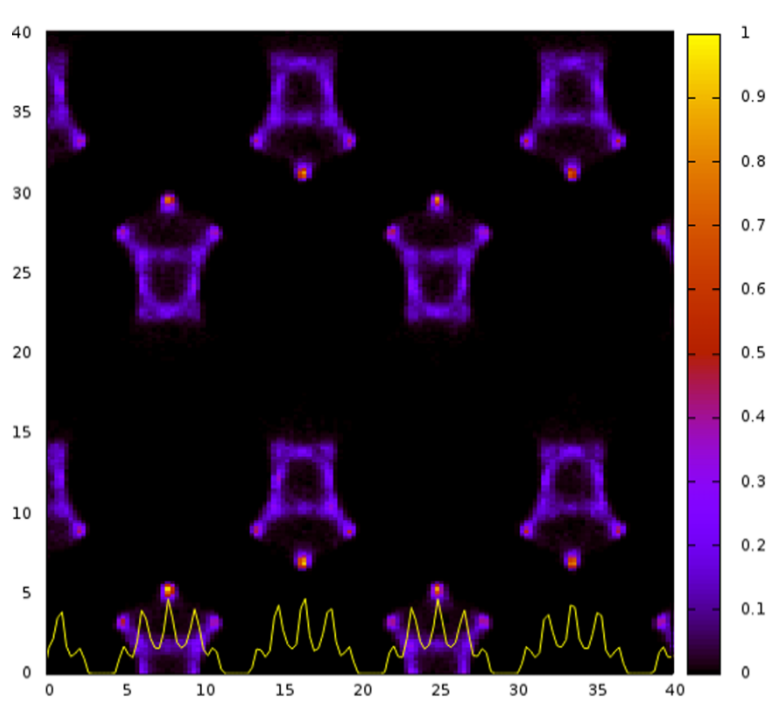

(a)

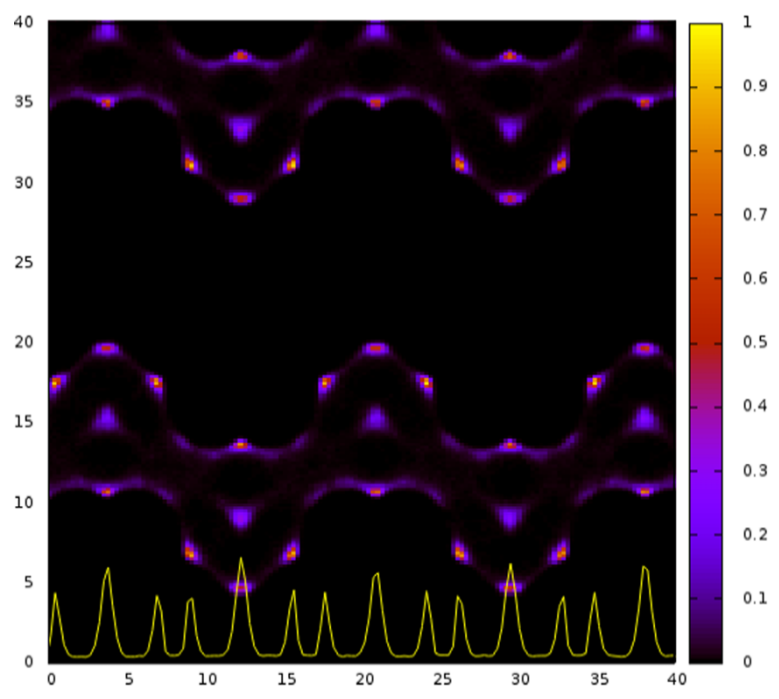

(c)

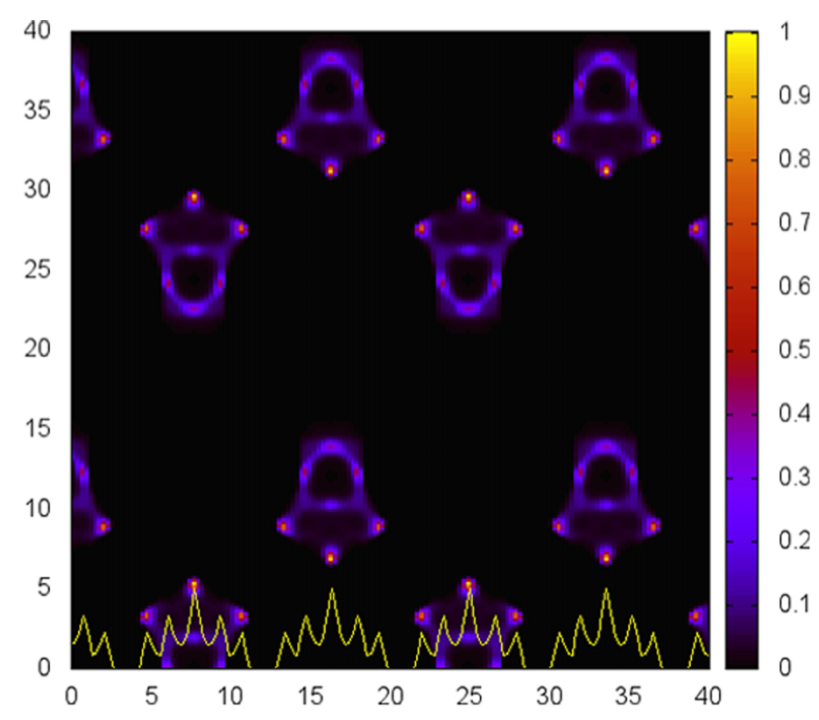

(b)

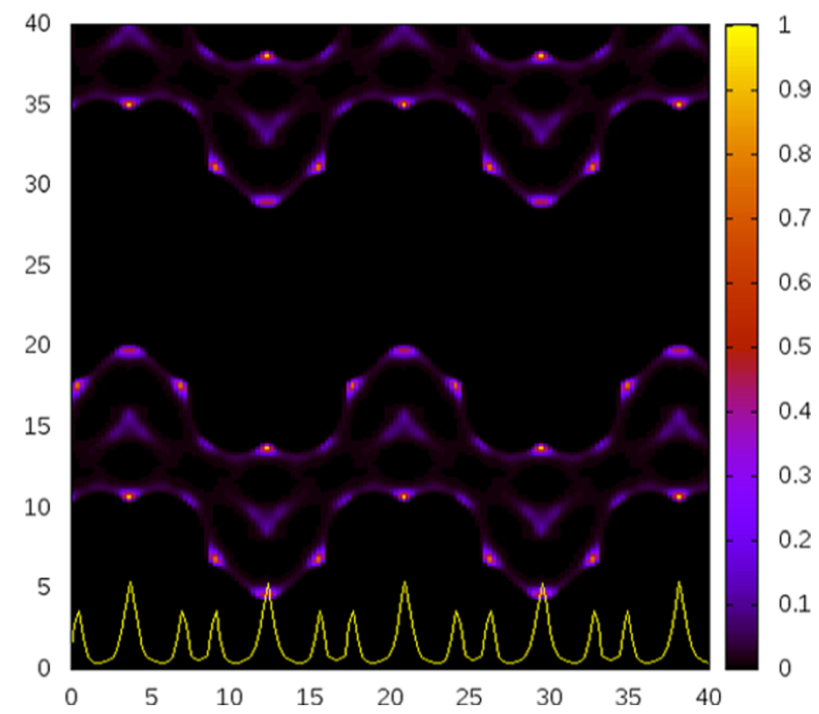

(d)

FIG. 13. Scaled fluid density distribution $\rho_{1}(x, y, z) / \rho^{\max }$ of adsorbed Ar atoms into a FAU zeolite corresponding to a slab of $1.5 \AA$ around the planes 110 (upper graphs) and $\overline{1} 10$ (lower graphs), as computed from the 3DOZ-HNC equation and MD simulations for $T=77 \mathrm{~K}$ and $104 \mathrm{Ar} / \mathrm{u} . \mathrm{c}$. Density profile projections on the diagonal axis of the $x y$ plane defined by $y=x$ or $y=L_{x}-x$ for a slab of $1.5 \AA$ thickness are illustrated by yellow curves and arbitrarily normalized to ease the visibility of the graphs. $\rho^{\max }$ is chosen as the maximum value of $\rho_{1}(x, y, z)$ - both in the 3DOZ and MD results - so as to normalize the color scale of the plot. (a) $\operatorname{MD} \rho_{1}(x, x, z) / \rho^{\max }$, (b) $3 \mathrm{DOZ} \rho_{1}(x, x, z) / \rho^{\max }$, (c) $\operatorname{MD} \rho_{1}\left(x, L_{x}-x, z\right) / \rho^{\max }$, (d) $3 \mathrm{DOZ} \rho_{1}\left(x, L_{x}-x, z\right) / \rho^{\max }$. 


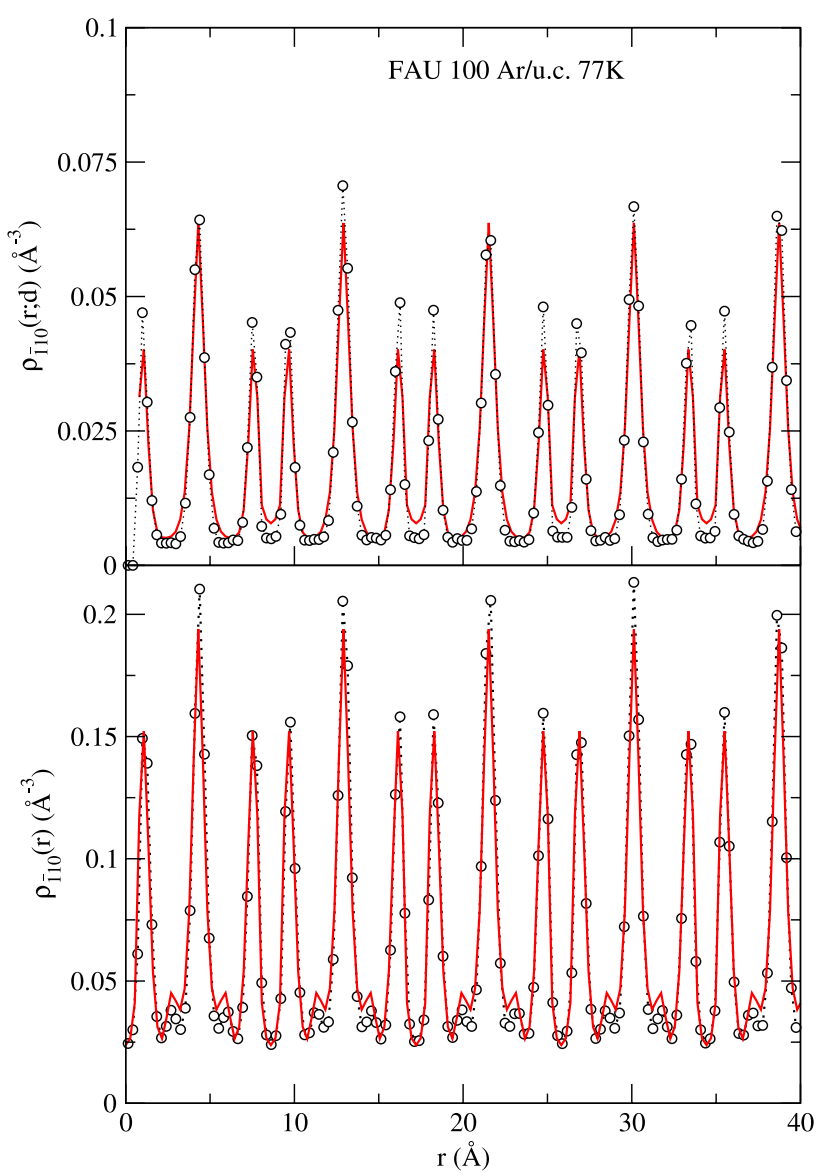

FIG. 14. Density profile of Ar atoms for a $1.5 \AA$ thick slab around the $\hbar 110$ plane, $\rho_{\hbar 110}(r ; d)$, and integrated over the $x y$ plane in the 110 -direction, $\rho_{\hbar 110}(r)$, for a load of $104 \mathrm{Ar}$ atoms adsorbed into a FAU zeolite at $77 \mathrm{~K}$ obtained from the 3DOZ-HNC approximation (solid curve) and computer simulation (circles). The $r$ represents the distance to the origin across the diagonal of the $x y$-plane.

planes. In all cases, we observe here an excellent agreement between the theory and the simulation for all the details of the profiles.

Finally, we take now a look at the results for Ar adsorbed into faujasite for a load of $104 \mathrm{Ar}$ atoms/u.c. and $77 \mathrm{~K}$. In Figure 13, we plot the density maps corresponding to slabs centered on the relevant planes for this framework, which are now the 110 and $\overline{1} 10$, orthogonal and parallel to the channels. The agreement is again fairly good, and one might even speculate that some discrepancies are due to the limited sampling of the MD run, e.g., some areas of the MD profile present a much starker contrast, which we have seen tends to become blurred as the sampling is increased. The corresponding cumulative density profile and the profile within a $1.5 \AA$ thick slab centered around the 110 plane are plotted in Figure 14. The overall performance of the theory is once more good with minor discrepancies in the heights of the peaks.

Finally, as an illustration of the three dimensional picture of the adsorbed fluid, we present in Figure 15 the three dimensional density isosurfaces using reference values like those of Figure 9. One can see now that for the FAU framework, high adsorbate concentrations can be found equally scattered along the channels and intersections.

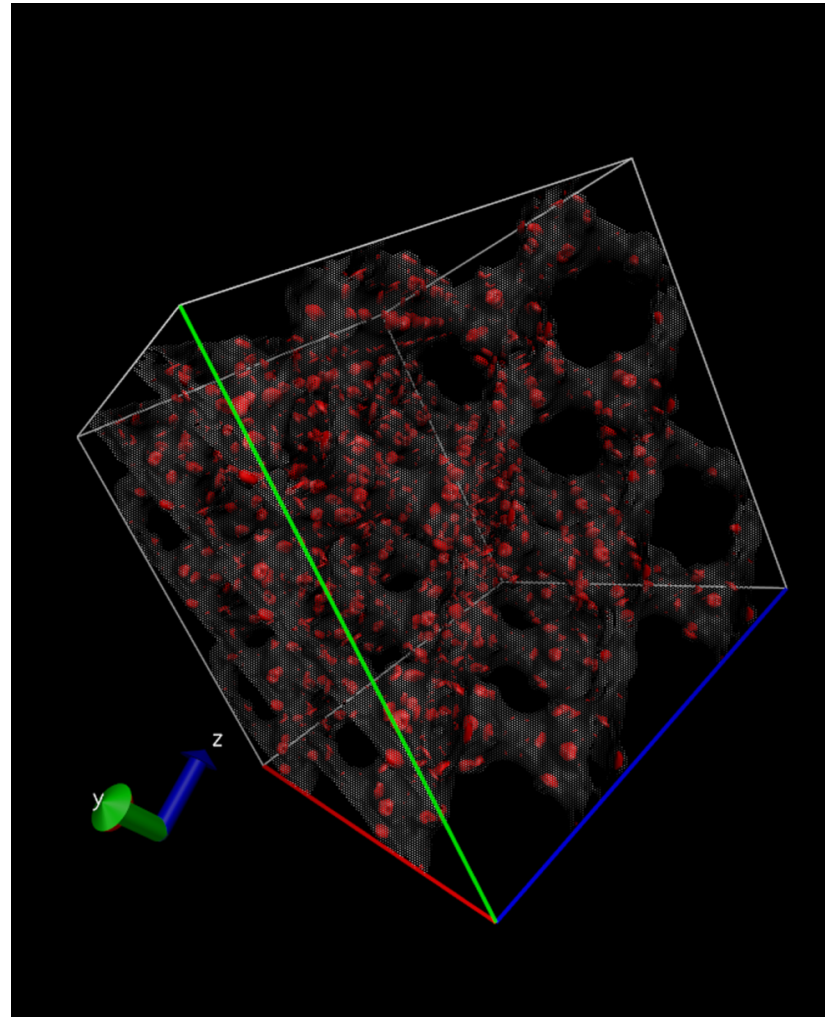

FIG. 15. Three-dimensional density distribution of Ar atoms for a load of $104 \mathrm{Ar}$ atoms adsorbed into a FAU zeolite at $77 \mathrm{~K}$ obtained from the 3DOZHNC approximation plotted by means of isosurfaces defined by $\rho_{1}(x, y, z)$ $=0.005 \AA^{-3}$ (glassy surface) and $0.058 \AA^{-3}$ (red).

\section{CONCLUSIONS}

In summary, we have presented the solution of the 3DOZHNC equation for model systems of Ar adsorbed into MEL, MFI, and FAU zeolites. Fluid-fluid direct correlation functions were approximated using the ROZ-HNC equations using as matrix-matrix correlations those calculated from the zeolite structure factors. The average fluid-fluid and fluid-zeolite structure provided by the ROZ-HNC using a one-component (oxygen atoms) matrix turned out to be qualitatively correct, particularly in the case of MFI and MEL (tight confinement). The lower quality of the results for FAU suggests that given the large diameter of this zeolite's channels, a better approximation might be achieved using either DFT or an inhomogeneous $\mathrm{OZ}$ integral equation for fluids in cylindrical pores. Interestingly, the adsorption isotherms obtained from the one component ROZ-HNC reproduce well the saturation limits of the zeolites and much better than those obtained using a two component matrix. Using the ROZ-HNC fluid-fluid correlations, the 3DOZ-HNC was solved for the three systems of interest, but convergence difficulties were found for the lowest temperature, $77 \mathrm{~K}$ under conditions of tight confinement (MEL framework). At higher temperatures/lower loads or for low temperature adsorption in faujasite, the theoretical results agree remarkably well with the simulation and provide an accurate three dimensional picture of the fluid distribution with a much higher resolution. Additionally, we have shown that the averaged fluid-adsorbate distribution function can also be accurately computed from the $3 \mathrm{DOZ}$ equation, outperforming 
the ROZ-HNC results for this structural quantity. The fact that the 3DOZ-HNC incorporates explicitly many body effects from the adsorbate-matrix interaction explains the better accuracy of this approach.

Work is planned on the investigation of alternative closures that can bypass the low temperature convergence difficulties found under conditions of tight confinement. We also plan to explore the use of DFT to obtain better approximations for fluid-fluid correlations. The study of confined mixtures and molecular fluids is currently under way.

\section{ACKNOWLEDGMENTS}

The authors acknowledge the support from the Dirección General de Investigación Científica y Técnica under Grant No. FIS2013-47350-C5-4-R. C.B. and V.S.G. acknowledge support from the Ministerio de Economía y Competitividad and the CSIC in the form of FPI and JAEPre fellowships, respectively.

${ }^{1}$ J. S. Perkyns, G. C. Lynch, J. J. Howard, and B. M. Pettitt, J. Chem. Phys. 132, 064106 (2010).

${ }^{2}$ D. Beglov and B. Roux, J. Chem. Phys. 103, 360 (1995).

${ }^{3}$ A. Kovalenko and F. Hirata, Chem. Phys. Lett. 290, 237 (1998).

${ }^{4}$ D. Henderson, F. Abraham, and J. Barker, Mol. Phys. 31, 1291 (1976)

${ }^{5}$ T. V. Ramakrishnan and M. Yussouff, Phys. Rev. B 19, 2775 (1979).

${ }^{6}$ T. Munakata and K. Kim, J. Chem. Phys. 113, 3975 (2000).

${ }^{7}$ K. Kim and T. Munakata, J. Chem. Phys. 117, 277 (2002).

${ }^{8}$ D. Beglov and B. Roux, J. Chem. Phys. 104, 8678 (1996).

${ }^{9}$ D. Beglov and B. Roux, J. Phys. Chem. B 101, 7821 (1997).

${ }^{10}$ Q. Du, D. Beglov, and B. Roux, J. Phys. Chem. B 104, 796-805 (2000).

${ }^{11}$ C. M. Cortis, P. J. Rossky, and R. A. Friesner, J. Chem. Phys. 107, 6400 (1997).

${ }^{12}$ A. Kovalenko and F. Hirata, J. Chem. Phys. 110, 10095 (1999).

${ }^{13}$ B. C. Wood, S. Y. Bhide, D. Dutta, V. S. Kandagal, A. D. Pathak, S. N. Punnathanam, K. G. Ayappa, and S. Narasimhan, J. Chem. Phys. 137, 054702 (2012).
${ }^{14}$ M. Boronat, C. Martínez-Sánchez, D. Law, and A. Corma, J. Am. Chem. Soc. 130, 16316-16323 (2008).

${ }^{15}$ M. Grahn, A. Holmgren, and J. Hedlund, J. Phys. Chem. C 112, 7717 (2008).

${ }^{16}$ C. A. Fyfe, A. C. Diaz, H. Grondey, A. R. Lewis, and H. Förster, J. Am. Chem. Soc. 127, 7543 (2005).

${ }^{17}$ P. L. Llewellyn, J. P. Coulomb, Y. Grillet, J. Patarin, H. Lauter, H. Reichert, and J. Rouquerol, Langmuir 9, 1846-1851 (1993).

${ }^{18}$ N. Floquet, J. P. Coulomb, J. P. Bellat, J. M. Simon, G. Weber, and G. Andre, J. Phys. Chem. C 111, 18182-18188 (2007).

${ }^{19}$ E. Lomba, C. Bores, and G. Kahl, J. Chem. Phys. 141, 164704 (2014).

${ }^{20}$ W. G. Madden and E. D. Glandt, J. Stat. Phys. 51, 537 (1988).

${ }^{21}$ J. A. Given and G. Stell, J. Chem. Phys. 97, 4573 (1992).

${ }^{22}$ E. Lomba, J. A. Given, G. Stell, J. J. Weis, and D. Levesque, Phys. Rev. E 48, 233 (1993).

${ }^{23}$ D. M. Bibby, N. B. Milestone, and L. P. Aldridge, Nature 280, 664 (1979).

${ }^{24}$ P. I. Ravikovitch and A. V. Neimark, Langmuir 22, 11171-11179 (2006).

${ }^{25}$ E. García-Pérez, J. B. Parra, C. O. Ania, D. Dubbeldam, T. J. H. Vlugt, J. M. Castillo, P. J. Merkling, and S. Calero, J. Phys. Chem. C 112, 9976-9979 (2008).

${ }^{26}$ D. H. Olson, G. T. Kokotailo, S. L. Lawton, and W. Meier, J. Phys. Chem. 85, 2238 (1981).

${ }^{27}$ O. Terasaki, T. Ohsuna, H. Sakuma, D. Watanabe, Y. Nakagawa, and R. C. Medrud, Chem. Mater. 8, 463 (1996).

${ }^{28}$ C. Baerlocher, L. McCusker, and D. Olson, Atlas of Zeolite Framework Types, 6th ed. (Elsevier, Amsterdam, The Netherlands, 2007).

${ }^{29}$ E. Lomba and J.-J. Weis, J. Chem. Phys. 132, 104705 (2010).

${ }^{30}$ E. Anderson, Z. Bai, C. Bischof, S. Blackford, J. Demmel, J. Dongarra, J. Du Croz, A. Greenbaum, S. Hammarling, A. McKenney et al., LAPACK Users' Guide, 3rd ed. (Society for Industrial and Applied Mathematics, Philadelphia, PA, 1999), ISBN: 0-89871-447-8 (paperback).

${ }^{31}$ B. Hribar, V. Vlachy, and O. Pizio, J. Phys. Chem. B 105, 4727-4734 (2001).

${ }^{32}$ L. L. Lee, J. Chem. Phys. 97, 8606 (1992).

${ }^{33}$ M.-J. Fernaud, E. Lomba, and L. L. Lee, J. Chem. Phys. 111, 10275 (1999).

${ }^{34} \mathrm{~J}$. Percus, in The Equilibrium Theory of Classical Fluids, edited by H. L. Frisch and J. L. Lebowitz (W. A. Benjamin, New York, 1964).

${ }^{35}$ M. Frigo and S. G. Johnson, Proc. IEEE 93, 216 (2005).

${ }^{36}$ R. Q. Snurr, A. T. Bell, and D. N. Theodorou, J. Phys. Chem. 97, 13742-13752 (1993).

${ }^{37}$ V. Sánchez-Gil, E. G. Noya, J. M. Guil, E. Lomba, and S. Valencia, “Adsorption of argon on pure silica MEL. Volumetric experiments and grand canonical Monte Carlo simulations," (to be published).

${ }^{38}$ A. Mellergaard and R. McGreevy, Chem. Phys. 261, 267 (2000). 